\title{
CONSERVED ROLE OF DROSOPHILA MELANOGASTER FOXP IN MOTOR COORDINATION AND COURTSHIP SONG
}

\author{
A Dissertation \\ Presented to the Faculty of the Graduate School \\ of Cornell University \\ In Partial Fulfillment of the Requirements for the Degree of \\ Doctor of Philosophy
}

by

K risty J o Lawton

J anuary 2014 
(c) 2014 K risty J o Lawton 


\title{
CONSERVED ROLE OF DROSOPHILA MELANOGASTER FOXP IN MOTOR COORDINATION AND COURTSHIP SONG
}

\author{
K risty Jo Lawton, Ph. D. \\ Cornell University 2014
}

FoxP2 is a transcription factor underlying a severe human speech and language disorder. Research on this gene in vertebrate model organisms such as mouse and zebra finch indicated that it is highly conserved between species and strongly expressed in motor related brain structures. Although these studies implicated FoxP2 in development of motor control regions, the precise mechanisms are not understood. This work is the first to explore the functional role of FoxP2's D rosophila homolog known as FoxP. We characterize the behavioral importance of this gene in flies using FoxP specific RNA interference throughout development, which impairs several types of behavior, with males more strongly affected. We also used a temperature sensitive UAS-Shibire line to disrupt FoxP neuron function in adulthood and we saw dramatic effects on motor coordination. Our work also provides the first visualization of FoxP expression in the fly brain. We see a small number of symmetrically expressed FoxP neurons in clusters throughout the brain, but no obvious difference between males and females. There is also strong FoxP expression in the protocerebral bridge within the central complex, which is essential for higher level locomotion control and is thought to be homologous to the vertebrate basal ganglia. FoxP2 in humans is also highly expressed in the basal ganglia, specifically in areas important for motor coordination. 
These results indicate a conserved functional homology of this gene between invertebrates and vertebrates, both in terms of behavioral effects on motor coordination as well as expression pattern. We also disrupted FoxP neurons throughout pupal stages and discover that eclosion behavior is abolished, indicating a possible role of FoxP in development during pupation. Thus we propose to establish D rosophila as a model to study this crucial speech disease gene. We believe this work will contribute to further understanding of the importance of FoxP transcription factors in humans, as well as provide further support for the idea of deep homology between the invertebrate and vertebrate brain. 


\section{BIOGRAPHICAL SKETCH}

Kristy was born in Portland, Oregon. She lived in rural areas in Oregon for her entire childhood and grew up surrounded by nature. Much of her time was spent outside observing and interacting with a wide variety of organisms, where she nurtured a fondness for animals and a strong curiosity for living creatures. Her love of animals continued into high school where she worked at a veterinary clinic, established her own highly successful pet-sitting business, and worked at an animal shelter. While she briefly considered a career in veterinary medicine, she had a greater urge to understand animal behavior.

For her undergraduate degree, she attended the University of Oregon in Eugene and pursued her interests in biology. In her sophomore year she was offered an undergraduate lab research position in the neuroscience lab of Shawn Lockery, where she studied the neural basis of locomotion in the nematode $C$. elegans. After graduation she moved to Japan to teach English for six months before returning to work in the Lockery lab to continue research full-time. Due to her interest in neuroscience and animal behavior, she joined the PhD program in the Department of Neurobiology and B ehavior at Cornell U niversity in Ithaca, $\mathrm{New}$ Y ork. Her interest in understanding the mechanisms underlying behavior drew her to study the genetic basis of behavior in the fruit fly Drosophila melanogaster in the lab of David Deitcher where she learned valuable skills for a career in neurobiology. 
For Dezzie 


\section{ACKNOWLEDGMENTS}

M any thanks to my advisor David Deitcher. His excellent mentoring, technical training, and positive personality made my research experience immensely fulfilling. I also appreciate the support and feedback from my committee members R on Hoy, Joe Fetcho, and A ndy Bass. I'd like to extend my thanks to other members of my lab both past and present, including Hannah Kim, Cassandravictoria Innocent, and Michael Huang, all of whom provided scientific support and friendship. I would also like to extend my gratitude to the members of the Hoy lab for kindly sharing their equipment and expertise. The NBB administrative staff have also been immensely helpful and friendly and I can only hope to again encounter such wonderful people in my future workplaces.

My parents, family, and friends back home deserve infinite thanks for their emotional support, wise advice, and constant encouragement.

I am endlessly thankful for the invaluable friends I have made while in Ithaca. I am so fortunate to have met every single one of them. Regardless of where everyone scatters around the country and the world, the relationships I've formed here have shaped me as a person and will continue to influence me in the future.

I'm especially grateful to my old friend Steven B. A ugustine for his voluntary assistance in developing the peak detection program for courtship song analysis.

It was a joy having the opportunity to mentor Cornell undergraduates Lisa Lema, Crystal Grant, Nipun B handari, and Taryn W assmer and I want to acknowledge them all for their assistance with data collection and their bright personalities in the 
lab.

This project was supported in part by the $\mathrm{N}$ ational Institutes of Health Cellular and M olecular Pre-doctoral Training Grant 2T32GM 007469, and from the Cornell University Dept. of Neurobiology and Behavior Student Research Grant in A nimal Behavior. 


\section{TABLE OF CONTENTS}

Biographical sketch

Dedication

A cknowledgements

Table of contents

List of figures

List of abbreviations

1. Introduction

$1.1 \mathrm{~A}$ brief overview

1.2 Understanding the neural basis of human speech and language

1.3 Development of an invertebrate model to study FoxP2

1.4 Perspectives

References

2. Conserved function of Drosophila melanogaster FoxP in behavior implies a crucial role in motor coordination 16

2.1 A bstract

2.2 Introduction

2.3 M ethods

2.4 Results

2.5 Discussion

References

3. Spatial and temporal analysis of FoxP in the nervous system

3.1 A bstract

3.2 Introduction

3.3 M ethods - 49

3.4 Results

3.5 Discussion

References

4. Summary and future directions

4.1 A brief overview

4.2 Characterizing the importance of D rosophila F ox $\bar{P}$ on fly behavior

4.3 Determining the location and timing of FoxP action

4.4 Future directions

References 
Figure 2.1 Protein sequence similarity betw een human transcription factor FOXP2 and the D rosophila melanogaster homolog FoxP

Figure 2.2 A male fly courting a female in a recording chamber

21

Figure 2.3 Confirming knockdown of FoxP expression 27

Figure 2.4 Effects of FoxP knockdown on male courtship behavior

Figure 2.5 Effects of female FoxP knockdown on male courtship behavior

Figure 2.6 Effects of FoxP knockdown on courtship song

Figure 2.7 Effects of FoxP knockdown on locomotion and flight

Figure 2.8 Walking behavior after disrupting neurotransmission in FoxP expressing cells

Figure 2.9 Quantification of average activity level after acute disruption 38 of FoxP expressing neurons

Figure 3.1 FoxP antibody staining in larva brain

52

Figure 3.2 Overlap of FoxP antibody staining and FoxP-GA L4 expression

Figure 3.3 Expression pattern of FoxP in the adult brain

54

Figure 3.4 Confirming if FoxP is in neurons

56

Figure 3.5 Confirming FoxP is not in glia

57

Figure 3.6 FoxP cell counts in FoxP>nls::GFP adult brains

58

Figure 3.7 Disruption of FoxP neurons during pupation prevents eclosion 


\section{LIST OF ABBREVIATIONS}

SLI Specific language impairment

DVD Developmental verbal dyspraxia

$\mathrm{Cl} \quad$ Courtship index

$\mathrm{CL} \quad$ Copulation latency

IPI Inter-pulse-interval

FI Flight index

DAMS Drosophila automated monitoring system

CX Central complex

PB Protocerebral bridge

BG Basal ganglia

CNS Central nervous system

GFP Green fluorescent protein

RNAi RNA interference

UAS Upstream activator sequence

Shi $^{\text {is }} \quad$ Temperature sensitive shibire

BLAST Basic local al ignment sequence tool

M RI M agnetic resonance imaging

fM RI Functional magnetic resonance imaging 


\section{CHAPTER 1}

\section{INTRODUCTION}

\subsection{B rief Overview}

Human language is an integral part of society. There are many cases of impaired vocal communication that negatively affect people throughout their lives. Understanding how the nervous system controls this uniquely complicated behavior allows for potential prevention and treatment of speech and language disorders and provides insight into how this ability developed over evolutionary time. Exploration of the link between genes and neural development is crucial to elucidate the underlying causes of these debilitating language disorders. M odern genetic and molecular tools utilized in model organisms are extremely useful to determine the fundamental neural structure upon which speech and language skills are built.

\subsection{Understanding the neural basis of human speech and language}

\subsubsection{The early years of speech studies}

Human language is arguably one of the most important motor skills we have acquired as a species. This ability has greatly contributed to formation of societies and provided a means for passing knowledge and history to subsequent generations. Decades of past and ongoing studies are gaining insight into how this capability developed and an understanding of changes in the brain which differentiate complicated human speech abilities from the more rudimentary vocalizations of our closely related primate ancestors and other animals. Besides providing 
interesting answers to questions about evolution of vocal communication, these studies suggest possible solutions to situations where human language ability is impaired.

Speech and language disorders are relatively common and can often be identified early in childhood. Specific language impairment $(S L I)$ is a disorder characterized by language production and comprehension impairments without other complications such as autism or sensory deficits. Several subtypes have been described, each with highly varied clinical manifestations (Schwartz 2009). One study estimated the prevalence rate of SLI in Englishspeaking A merican kindergarteners at approximately $8 \%$ and $6 \%$ for boys and girls respectively (Tomblin et al. 1997). Given these relatively high incidence rates and the strong social and educational importance of verbal communication skills, understanding the developmental and neural basis of speech and language disorders is of vital importance.

Early clues to speech and language related brain regions relied on lesion studies in individuals with localized brain injuries from stroke or accident. A classic example is the identification in the late 1800s of two distinct language-related regions of the cerebral cortex Broca's area which is vital for language production and Wernicke's area necessary for comprehension. Although somewhat crude, discovery of these areas informed early researchers that specific locations in the brain are specialized for language related tasks. Later imaging technology such as functional magnetic resonance imaging ( $\mathrm{fMRI}$ ) allowed for detailed analysis of active brain regions during language tasks. The results of this technique implicated a wider variety of brain regions involved in specific aspects of language (reviewed in Conti-Ramsden and Durkin 2012). 
Although language deficits may be due in part to environmental causes (given correlations of higher incidence rates in certain groups such as low-income uneducated families), lineage, twin, and linkage studies have pointed towards genetic influences on development of speech and language (reviewed in Gopnik and Crago 1991 and Bishop 2001).

\subsubsection{The KE family and $F$ oxP 2}

The clearest example of a genetic based language disorder is the case of a well-studied multigenerational pedigree in England known as the KE family. They exhibit a severe developmental verbal dyspraxia (DVD), which manifests as difficulty in complex speech production as well as language comprehension (Gopnik \& Crago 1991; V argha-K hadem et al. 1995). They exhibit orofacial-dyspraxia which impairs coordination of fine movement sequences in the speech production hardware of the face and mouth, but do not show any obvious issues with muscle weakness (Alcock et al. 2000). At the same time, they have a persistent difficulty with grammar and language comprehension (W atkins, Dronkers, and V argha-K hadem 2002). Importantly, this disease is a simple autosomal-dominant $\mathrm{M}$ endelian inheritance, indicating a direct genetic etiology from an individual gene. Linkage analysis narrowed the region of this gene to a small area on chromosome 7 (Fisher et al. 1998), and in $2001 \mathrm{Lai}$ et al. indentified the

underlying cause to be a single point mutation in the DNA binding domain of the FoxP2 gene, which is a member of a large winged-helix transcription factor family. Subsequently, several other unrelated individuals with language impairments were discovered to have deletions and truncations of FOX P2 (M acDermot et al. 2005; Feuk et al. 2006; Rice et al. 2011; Turner et al. 2013), and a few single nucleotide polymorphisms in the gene correlated with differential brain activation in language related areas during an fM RI task (Pinel et al. 2012). These discoveries 
provided the first direct genetic clue to language development and opened the possibility for a greater understanding of the developmental origins of language.

Identification of FOXP2 expression in fetal brains indicated FOXP2 may play a role in several key motor regions in the brain, including the cerebellum and areas of the basal ganglia, with especially strong expression in the striatum (Ferland et al. 2003; Lai et al. 2003). This region of the basal ganglia is known to be involved in motor coordination and has extensive connections with higher order cerebral function (M iddleton \& Strick 2000). Imaging studies in the KE family also showed relative size (W atkins and V argha-K hadem 2002) and fM RI activity changes (Liégeois et al. 2003) in similar brain regions.

\subsubsection{U tilizing animal models to understand the role of $F$ oxP 2}

Given the constraints of studying humans, animal models are often useful for gaining a deeper understanding of genes involved in human disorders. Speech and language as we know it may be unique to humans, but many other animals exhibit complex sound production for social communication. It was quickly discovered that FoxP2 is very highly conserved across vertebrate species. For example, in mice the FoxP2 homolog shows only three differing amino acids in the functional regions of the protein (Enard et al. 2002) and it is expressed in similar brain regions between the two species. A gain, FoxP2 was highly expressed in a variety of motor related areas, including the striatum (Ferland et al. 2003).

Although the brain structure is somewhat different, a bird model organism - the zebra finch -provides a useful model for studying FoxP2, as they also exhibit complex learned vocalizations in the form of song. Zebra finch FoxP2 sequence is also highly conserved and is expressed in several overlapping brain regions as in humans, including the striatal region known 
as A rea $X$ (Teramitsu et al. 2004). A rea $X$ is a specific region of the bird striatum known to be an integral part of birdsong learning and production (reviewed in Bolhuis, Okanoya, and Scharff 2010). Suppression of FoxP2 expression in this area of the finch brain during the critical song learning period greatly impaired adult song (Haesler et al. 2007). The birds were still able to form an adult crystallized song, but syllables within the song were disordered or incorrect in ways reminiscent of speech errors in affected members of the KE family. Other studies showed a variety of modulations of FoxP2 expression in A rea X during juvenile song learning and indicate that FoxP2 continues to play a role in adult song production (Teramitsu \& White 2006; Teramitsu et al. 2010; Haesler et al. 2007; Miller et al. 2010; Thompson et al. 2013). These studies provide the closest approximation to complex learned vocalizations in a non-human animal. Zebra finch FoxP2 also strongly implicates the striatum as an area of vital FoxP2 action, but this model provides limitations as a wide variety of genetic tools are still lacking. Thus much work on FoxP2 shifted to the mouse - another vertebrate model with a wider selection of genetic tools.

As mentioned earlier, mice and human FoxP2 protein is almost identical in sequence (Enard et al. 2002) and they share expression patterns in similar brain regions, with strong expression in the basal ganglia (Ferland et al. 2003; Lai et al. 2003). Although mice are not known to have learned complex vocalizations, the pups do emit multiple types of vocalizations to elicit maternal care (Branchi et al. 2001). In mouse behavioral studies with alterations of normal FoxP2 expression, impairments in pup cries and motor coordination are apparent. Homozygous FoxP2 mutants and knockouts show delayed growth and die at three weeks of age. For heterozygotes development appears normal, but some reports indicate disrupted elements of pup cries (Shu et al. 2005; Fujita et al. 2008). In addition to vocalization assays, other motor 
behaviors were tested in heterozygotes which showed deficits in motor coordination in pups and adult mice (Shu et al. 2005; Fujita et al. 2008; Groszer et al. 2008). Further exploration of the brains of these mice indicated structural abnormalities in the cerebellum (Scharff \& Haesler 2005), and changes in synaptic plasticity in both the cerebellum and basal ganglia (G roszer et al. 2008; French et al. 2012). A humanized version of FoxP2 in mice has opposite effects on plasticity in the basal ganglia (Enard et al. 2009; Reimers-Kipping et al. 2010).These results indicate a more generalized but highly conserved role of this gene in fine motor control, with several of these studies implicating possible important roles for the basal ganglia.

The FOX gene family is extensive, but all are transcription factors which share a similar winged-helix DNA binding domain, and the majority act as transcriptional activators (Stroud et al. 2006). M any FOX genes are involved in human diseases and neural development (Lehmann et al. 2003). Studies looking more specifically at the molecular properties of the FoxP2 protein indicated that it is usually a transcriptional repressor (Vernes et al. 2007), and requires dimerization with itself or other FoxP family members for normal function (Li et al. 2004). Chromatin immunoprecipitation assays combined with microarrays identified an extensive list of potential FoxP2 regulated genes, many of which are thought to be involved in development of neural networks (Vernes et al. 2007; Spiteri et al. 2007; Vernes et al. 2011). Focus on one of these, CNTNAP2, found a correlation with language processing and autism (Peter et al. 2011; Kos et al. 2012; Poot et al. 2010), but the majority of others remain unexplored. As yet, many questions remain unanswered about the details of FoxP2 actions in the nervous system and its precise role in human speech. 


\subsection{Development of an invertebrate model to study F oxP2}

1.3.1. The utility of the genetic model organism D rosophila melanogaster

Looking beyond vertebrate limitations, the fruit fly Drosophila melanogaster is a longstanding model for genetic studies, with an extensive molecular toolkit allowing for a deeper exploration of the link between genes and behavior. $M$ any genes are highly conserved between flies and humans (approximately 70\% for known disease genes), and the fly has provided a great deal of information on form and function of key developmental genes essential for both invertebrates and vertebrates. An excellent example of this is the discovery of the homeobox (Hox) genes encoding for transcription factors responsible for patterning the anterior-posterior body axis in early embryos (MCGinnis \& Krumlauf 1992). The original FOX gene was discovered and named in Drosophila as well (Lai et al. 1991). In addition, despite the great differences in brain structure between insects and humans, there are several similar regions. This includes the olfactory glomeruli which are organized in a way that resembles the vertebrate olfactory system (Hildebrand \& Shepherd 1997) as well as the mushroom bodies, which are analogous to the vertebrate hippocampus, which are involved in learning and memory (Cayre et al. 2002). The central complex is also thought to be homologous to the basal ganglia and is important for motor coordination in both structures (Strausfeld \& Hirth 2013).

The fly also exhibits relatively complex behaviors requiring intricate motor coordination, many of which are social behaviors (Sokolowski 2010). Included among these is courtship, where the male repeats many steps in a stereotyped courtship sequence incorporating song production as a major component. M ales "sing" to females by vibrating a single wing to produce two different song types (Ewing 1983). Only the males sing and direct this attention to the 
females who exhibit a more limited set of behavior including running away from the pursuing male, rejection behaviors when the male attempts to copulate, and acceptance behavior leading to successful copulation. The two types of courtship song produced by the male are accomplished by a unilateral wing extension and vibration. Sine song is a simple hum and seen with less frequency than pulse song, which consists as a series of short pulses separated by a precisely regulated inter-pulse-interval (IPI) (Greenspan \& Ferveur 2000). This IPI varies between different Drosophila species and is suspected to serve as a method for females to distinguish males of their own species, and perhaps serve as some way to evaluate male fitness (Talyn \& Dowse 2004).

A defining feature of the fly model is the fully sequenced genome and an established suite of molecular tools available to manipulate gene expression. One powerful strategy is the GAL4/UAS system which is frequently used in Drosophila to drive expression of a gene-ofinterest in a specified cell type. The GAL4 is a transcriptional activator originally from yeast which can be expressed under promoters for specific genes. GA L 4 binds to an upstream activator sequence (UAS) which transcribes a gene of interest. UAS lines are available to activate a variety of genetic tools, such as visualizing gene expression with reporter proteins, or targeted reduction of expression of a specified gene via RNA interference. A combination of these techniques is used in the work described in the following chapters.

\subsubsection{The fly F oxP 2 homolog}

Most importantly for this dissertation work is the recently discovered Drosophila homolog of FoxP2, known as FoxP, which is highly conserved in amino acid sequence with 
especially high conservation in the functional DNA binding domain. As in vertebrates, FoxP is also strongly expressed in the nervous system from early in development (Lee \& Frasch 2004; Santos et al. 2011; Chintapalli et al. 2007). One recent study determined that FoxP has two splice variants, with both expressed in the nervous system and one also in hemocytes (Santos et al. 2011), but the function of this gene in flies remained unexplored. At the start of this project, FoxP was still unnamed and known only by number (CG 16899). The work presented here provides the first characterization of Drosophila FoxP's behavioral function, expression pattern, and importance in developmental timing, and establishes a new model organism to contribute understanding of this well-conserved and vital transcription factor across organisms.

\subsection{Perspectives}

This chapter provided a review of the research attempting to understand the genetics and neural development underlying speech and language production, how the transcription factor FoxP2 ties into this understanding, and the potential utility of the fruit fly Drosophila melanogaster as a model system to explore the role of FoxP2. Chapter 2 establishes the behavioral importance of FoxP in flies. Chapter 3 identifies the specific brain regions expressing FoxP as well as the crucial timing of action of this gene. Chapter 4 provides a summary and discussion of the previous chapters, as well as future directions. 


\section{REFERENCES}

Alcock, K.J. et al., 2000. Oral dyspraxia in inherited speech and language impairment and acquired dysphasia. Brain and language, 75(1), pp.17-33.

Bishop, D. V, 2001. Genetic and environmental risks for specific language impairment in children. Philosophical transactions of the Royal Society of London. Series B, Biological sciences, 356(1407), pp.369-80.

Bolhuis, J.J., Okanoya, K. \& Scharff, C., 2010. Twitter evolution: converging mechanisms in birdsong and human speech. Nature Reviews Neuroscience, 11(11), pp.747-759.

Branchi, I., Santucci, D. \& Alleva, E., 2001. Ultrasonic vocalisation emitted by infant rodents: a tool for assessment of neurobehavioural development. Behavioural brain research, 125(12), pp.49-56.

Cayre, M. et al., 2002. The common properties of neurogenesis in the adult brain: from invertebrates to vertebrates. Comparative biochemistry and physiology. Part B, Biochemistry \& molecular biology, 132(1), pp.1-15.

Chintapalli, V.R., Wang, J. \& Dow, J.A.T., 2007. Using FlyA tlas to identify better Drosophila melanogaster models of human disease. Nature genetics, 39(6), pp.715-20.

Conti-Ramsden, G. \& Durkin, K ., 2012. Language development and assessment in the preschool period. Neuropsychology review, 22(4), pp.384-401.

Enard, W. et al., 2009. A humanized version of Foxp2 affects cortico-basal ganglia circuits in mice. Cell, 137(5), pp.961-71.

Enard, W. et al., 2002. M olecular evolution of FOX P2, a gene involved in speech and language. Nature, 418(6900), pp.869-72.

Ewing, A., 1983. Functional aspects of Drosophila courtship. Biological Reviews, pp.275-292.

Ferland, R.J. et al., 2003. Characterization of Foxp2 and Foxp1 mRNA and protein in the developing and mature brain. The J ournal of comparative neurology, 460(2), pp.266-79.

Feuk, L. et al., 2006. A bsence of a paternally inherited FOXP2 gene in developmental verbal dyspraxia. American journal of human genetics, 79(5), pp.965-72.

Fisher, S.E. et al., 1998. Localisation of a gene implicated in a severe speech and language disorder. Nature genetics, 18(2), pp.168-70.

French, C.A. et al., 2012. An aetiological Foxp2 mutation causes aberrant striatal activity and alters plasticity during skill learning. M olecular psychiatry, 17(11), pp.1077-85. 
Fujita, E. et al., 2008. Ultrasonic vocalization impairment of Foxp2 (R552H) knockin mice related to speech-language disorder and abnormality of Purkinje cells. Proceedings of the National Academy of Sciences of the U nited States of America, 105(8), pp.3117-22.

Gopnik, M . \& Crago, M.B., 1991. Familial aggregation of a developmental language disorder. Cognition, 39(1), pp.1-50.

Greenspan, R.J . \& Ferveur, J.F., 2000. Courtship in Drosophila. Annual review of genetics, 34, pp.205-232.

Groszer, M. et al., 2008. Impaired synaptic plasticity and motor learning in mice with a point mutation implicated in human speech deficits. Current biology/: CB, 18(5), pp.354-62.

Haesler, S. et al., 2007. Incomplete and inaccurate vocal imitation after knockdown of FoxP2 in songbird basal ganglia nucleus A rea X. PL oS biology, 5(12), p.e321.

Hildebrand, J.G. \& Shepherd, G.M., 1997. M echanisms of olfactory discrimination: converging evidence for common principles across phyla. Annual review of neuroscience, 20, pp.595631.

K os, M. et al., 2012. CNTNAP2 and Language Processing in Healthy Individuals as M easured with ERPs. PloS one, 7(10), p.e46995.

Lai, C.S. et al., 2001. A forkhead-domain gene is mutated in a severe speech and language disorder. Nature, 413(6855), pp.519-23.

Lai, C.S.L. et al., 2003. FOXP2 expression during brain development coincides with adult sites of pathology in a severe speech and language disorder. Brain/: a journal of neurology, 126(Pt 11), pp.2455-62.

Lai, E. et al., 1991. Hepatocyte nuclear factor 3 al pha belongs to a gene family in mammals that is homologous to the Drosophila homeotic gene fork head. Genes \& Development, 5(3), pp.416-427.

Lee, H. \& Frasch, M., 2004. Survey of forkhead domain encoding genes in the Drosophila genome: Classification and embryonic expression patterns. Developmental dynamics/: an official publication of the American Association of Anatomists, 229(2), pp.357-66.

Lehmann, 0., Sowden, J. \& Carlsson, P., 2003. Fox's in development and disease. TRE NDS in ..., 19, pp.339-344.

Li, S., W eidenfeld, J. \& M orrisey, E.E., 2004. Transcriptional and DNA binding activity of the Foxp1/2/4 family is modulated by heterotypic and homotypic protein interactions. Molecular and cellular biology, 24(2), pp.809-22. 
Liégeois, F. et al., 2003. Language fM RI abnormalities associated with FOXP2 gene mutation. Nature neuroscience, 6(11), pp.1230-7.

MacDermot, K.D. et al., 2005. Identification of FOXP2 truncation as a novel cause of developmental speech and language deficits. American journal of human genetics, 76(6), pp.1074-80.

McGinnis, W. \& Krumlauf, R., 1992. Homeobox genes and axial patterning. Cell, 68(2), pp.283-302.

Middleton, F. a \& Strick, P.L., 2000. Basal ganglia output and cognition: evidence from anatomical, behavioral, and clinical studies. Brain and cognition, 42(2), pp.183-200.

M iller, J.E., Hilliard, A .T. \& W Wite, S. a, 2010. Song practice promotes acute vocal variability at a key stage of sensorimotor learning. P loS one, 5(1), p.e8592.

Peter, B. et al., 2011. Replication of CNTNA P2 association with nonword repetition and support for FOX P2 association with timed reading and motor activities in a dyslexia family sample. J Neurodevelop Disord, pp.39-49.

Pinel, P. et al., 2012. Genetic V ariants of FOXP2 and KIAA 0319/TTRAP/THEM 2 L ocus A re A ssociated with $A$ Itered B rain A ctivation in Distinct Language-Related R egions. J ournal of Neuroscience, 32(3), pp.817-825.

Poot, M. et al., 2010. Disruption of CNTNAP2 and additional structural genome changes in a boy with speech delay and autism spectrum disorder. Neurogenetics, 11(1), pp.81-9.

Reimers-Kipping, S. et al., 2010. Humanized Foxp2 specifically affects cortico-basal ganglia circuits. Neuroscience, pp.1-10.

Rice, G.M. et al., 2011. Phenotype of FOX P2 haploinsufficiency in a mother and son. American journal of medical genetics. Part $A$.

Santos, M .E. et al., 2011. A Iternative splicing and gene duplication in the evolution of the FoxP gene subfamily. Molecular biology and evolution, 28(1), pp.237-47.

Scharff, C. \& Haesler, S., 2005. An evolutionary perspective on FoxP2: strictly for the birds? Current opinion in neurobiology, 15(6), pp.694-703.

Shu, W. et al., 2005. A Itered ultrasonic vocalization in mice with a disruption in the Foxp2 gene. Proceedings of the National Academy of Sciences of the U nited States of America, 102(27), pp.9643-8.

Sokolowski, M .B., 2010. Social interactions in "simple" model systems. Neuron, 65(6), pp.78094. 
Spiteri, E. et al., 2007. Identification of the transcriptional targets of FOXP2, a gene linked to speech and language, in developing human brain. American journal of human genetics, 81(6), pp.1144-57.

Strausfeld, N.J \& \& Hirth, F., 2013. Deep homology of arthropod central complex and vertebrate basal ganglia. Science (N ew York, N.Y.), 340(6129), pp.157-61.

Stroud, J.C. et al., 2006. Structure of the forkhead domain of FOXP2 bound to DNA. Structure (London, England/: 1993), 14(1), pp.159-66.

Talyn, B.C. \& Dowse, H.B., 2004. The role of courtship song in sexual selection and species recognition by female Drosophila melanogaster. Animal B ehaviour, 68(5), pp.1165-1180.

Teramitsu, I. et al., 2004. Parallel FoxP1 and FoxP2 expression in songbird and human brain predicts functional interaction. The Journal of neuroscience/: the official journal of the Society for Neuroscience, 24(13), pp.3152-63.

Teramitsu, I. et al., 2010. Striatal FoxP2 is actively regulated during songbird sensorimotor learning. PloS one, 5(1), p.e8548.

Teramitsu, I. \& White, S.A., 2006. FoxP2 regulation during undirected singing in adult songbirds. The Journal of neurosciencel: the official journal of the Society for Neuroscience, 26(28), pp.7390-4.

Thompson, C.K. et al., 2013. Y oung and intense: FoxP2 immunoreactivity in A rea $\mathrm{X}$ varies with age, song stereotypy, and singing in male zebra finches. Frontiers in neural circuits, 7(February), p.24.

Tomblin, J.B. et al., 1997. Prevalence of specific language impairment in kindergarten children. J ournal of speech, language, and hearing research/: J SL HR, 40(6), pp.1245-60.

Turner, S.J. et al., 2013. Small intragenic deletion in FOXP2 associated with childhood apraxia of speech and dysarthria. American journal of medical genetics. Part A, pp.1-6.

V argha-K hadem, F. et al., 1995. Praxic and nonverbal cognitive deficits in a large family with a genetically transmitted speech and language disorder. P roceedings of the National Academy of Sciences of the U nited States of America, 92(3), pp.930-3.

Vernes, S.C. et al., 2011. Foxp2 Regulates Gene Networks Implicated in Neurite Outgrowth in the Developing B rain J. M. A key, ed. PLOS G enetics, 7(7), p.e1002145.

Vernes, S.C. et al., 2007. High-throughput analysis of promoter occupancy reveals direct neural targets of FOXP2, a gene mutated in speech and language disorders. American journal of human genetics, 81(6), pp.1232-50. 
Watkins, K.\& V argha-K hadem, F., 2002. M RI analysis of an inherited speech and language disorder: structural brain abnormalities. Brain, 125, pp.465-478.

Watkins, K .E., Dronkers, N.F. \& V argha-K hadem, F., 2002. B ehavioural analysis of an inherited speech and language disorder: comparison with acquired aphasia. Brain/: a journal of neurology, 125(Pt 3), pp.452-64. 


\section{CHAPTER 2}

\section{CONSERVED FUNCTION OF DROSOPHILA MELANOGASTER FOXP IN BEHAVIOR IMPLIES A CRUCIAL ROLE IN MOTOR COORDINATION}

\subsection{Abstract}

FoxP2 is a highly conserved vertebrate transcription factor known for its importance in human speech and language production. Disruption of FoxP2 in several vertebrate models indicates a conserved functional role for this gene in both sound production and motor coordination, but little is known about FoxP2's precise role in the nervous system. The recent discovery of the well-conserved Drosophila melanogaster homolog, FoxP, provides an opportunity to study the role of this crucial gene in an invertebrate model. We hypothesized that, like FoxP2, D rosophila FoxP is important for behaviors requiring fine motor coordination. We used targeted RNA interference to reduce expression of FoxP and assayed the effects on a variety of adult behaviors. Male flies with reduced FoxP expression exhibit decreased levels of courtship behavior, altered pulse-song structure, and sex-specific motor impairments in walking and flight. A cute disruption

of synaptic activity in FoxP expressing neurons using a temperature-sensitive shibire allele dramatically impaired motor coordination. Our results establish the necessity of this gene in motor coordination in an invertebrate model and suggest a functional homology with vertebrate FoxP2. 


\subsection{Introduction}

Language is a vital part of human social behavior. This complex trait is impaired in approximately $7 \%$ of the population and this deficit manifests in early childhood (Tomblin et al. 1997). Several studies indicate that deficits are largely due to complex genetic influences (Bishop 2006). Identifying the evolutionary origin and construction of the neural networks involved in acoustic communication requires an understanding of the underlying genetics, but until recently, specific genes have remained elusive.

FoxP2 is a vertebrate transcription factor known for its importance in speech and language production in humans. It's role in human behavior was originally discovered in a multigenerational family whose affected members have severe developmental verbal dyspraxia (DVD) throughout life, and underlying this deficit is a single point mutation in the DNA binding domain of FOXP2 ( $L$ ai et al. 2001). Since this discovery, independent mutations and truncations of FoxP2 have been linked to disorders with specific impairment in production of fluent speech (Lai et al. 2001; M acDermot et al. 2005; Zhao et al. 2010). A cross vertebrate models, FoxP2 is remarkably well conserved, both in amino acid sequence and brain expression patterns (Enard et al. 2002; Ferland et al. 2003; Haesler et al. 2004). For potential human language disease genes, animal models which show highly conserved form and function between species provide the means for a deeper understanding of the details of sound production.

FoxP2 effects on vocal production are not unique to humans. As a parallel to learned human speech, knockdown of FoxP2 in male zebra finch chicks during the critical song learning period significantly alters the structure of their crystallized adult song (Haesler et al. 2007). This result closely resembles grammatical impairments seen in humans, indicating that FoxP2 may play a conserved functional role in vocal production. In mice, a variety of F OXP 2 mutations and 
deletions have demonstrated effects on development and behavior. FoxP2 null mice are developmentally delayed and die within 3 weeks of birth, indicating a crucial role of FoxP2 in early postnatal life (Groszer et al. 2008; Fujita et al. 2008; Shu et al. 2005). In contrast, mice heterozygous for functional FoxP2 were developmentally normal but exhibited a variety of other deficits, such as a reduction in the amplitude of ultrasonic vocalizations (Gaub et al. 2010), abnormal synaptic plasticity, and deficits in motor skill learning (Groszer et al. 2008; K urt et al. 2012; French et al. 2012). From this variety of work in vertebrates, it is suggested that FoxP2 plays a role in fine motor control, which may have provided a neural substrate for development of complex vocalizations such as language (Fisher \& Scharff 2009). Despite these insights into the potentially conserved role of FoxP2 in sound production and fine motor control, the precise function of this gene remains poorly understood.

Recently, a gene in the fruit fly Drosophila melanogaster was identified as a closely related homolog to the vertebrate FoxP subfamily (Santos et al. 2011). The discovery of this invertebrate homolog in a genetically tractable organism such as Drosophila provides new possibilities for functional analysis and understanding of the evolutionary importance of the F oxP2 gene.

The results presented in this chapter provide the first functional characterization of the previously unexplored insect F oxP gene. I hypothesized that the fly FoxP would have conserved behavioral functions comparable to FOXP2. The rationale for this is that FoXP is highly similar to vertebrate FoxP2 in several ways. First, the protein sequence is well conserved between the two, especially in the DNA binding domain. This is shown clearly when using BLAST to align human FOXP2 to fly FoxP protein sequences (Fig. 1) (M arygold et al. 2013). 


\begin{tabular}{|c|c|c|}
\hline FOXP2 & 340 & $\begin{array}{l}\text { HTLYGHGVCKWPGCESICEDFGQFLKHLNNEHALDDRSTAQCRVQMQVVQQLEIQLSKER } \\
\begin{array}{lllll}\text { H L+ HG+C+WPGCE } & \text { ED } & \text { F+KHLN EH LDDRSTAQ RVQMQVV QLE L KER }\end{array}\end{array}$ \\
\hline CG16899 & 155 & HPLFAHGICRWPGCEMDLEDITSFVKHLNTEHGLDDRSTAÕARVQ๊MÕVVSQ̃LESHLQKER \\
\hline FOXP2 & 400 & ERLQAMMTHLHMRPSEPKPSP \\
\hline & & +RLQAMM HL++ \\
\hline CG16899 & 215 & DRLQAMMHHLYLSKQ----------LLSPTKIDRKDVPGREGKFCRSPLTVNSIGRPIRQ \\
\hline FOXP2 & 458 & 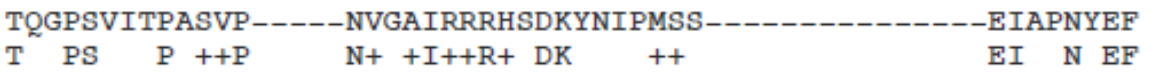 \\
\hline CG16899 & 265 & TNSPS---PLNLPMVNSTNLCSIKKRNHDKNTFS INGGLPYMLERAGLDVQQEIHRNREF \\
\hline FOXP2 & 498 & $\begin{array}{l}\text { YKNADVRPPFTYATLIRQAIMESSDRQLTLNEIYSWFTRTFAYFRRNAATWKNAV RHNLS } \\
\text { YKNADVRPPFTYA+LIRQAI++S D+QLTLNEIY+WF TF YFRRNAATWKNAVR HNLS }\end{array}$ \\
\hline CG16899 & 322 & YKNADVRPPFTYASLIRQAIIDSPDKQLTLNEIYNWFQNTFCYFRRNAATWKNAV BHNLS \\
\hline FOXP2 & 558 & $\begin{array}{l}\text { LHKCFVRVENVKGAVWTVDEVEYQKRRSQKITG---SPTLVKNIPTSLGYGAALN } \\
\text { LHKCF+RVENVKGAVWTVDE+E+ KRR Q }+\mathrm{G}+\mathrm{T} \text { N P }+\mathrm{Y} A+\mathrm{N}\end{array}$ \\
\hline CG16899 & 382 & LHKCFMRVENVKGAVWTVDEIEFYKRRPQRTAGIGNNLTGATNSPDT-NYFVAMN \\
\hline
\end{tabular}

\section{Figure 1. Protein sequence similarity between human transcription factor FOXP2 and the} Drosophila melanogaster homolog FoxP (C 16899). The top lines of text correspond to a portion of the amino acid sequence of the human FOXP2 protein. The bottom lines labeled CG16899 correspond to the Drosophila FoxP amino acid sequence. The middle lines indicate matching amino acids (letters) or conserved substitutions (+). The highlighted section marks the forkhead DNA binding domain, and the boxed arginine residue $(R)$ indicates the amino acid which contains the mutation in the human KE family with a severe speech disorder. 
Secondly, both are highly expressed in the nervous system from early in development (Chintapalli et al. 2007; Lee \& Frasch 2004; Santos et al. 2011). Lastly, fruit flies produce an acoustic social signal in the form of courtship song. This courtship sequence is a highly stereotyped and easily quantifiable sequence of body movements including singing, where the male extends and vibrates a single wing to produce songs (Fig. 2). If FoxP serves an analogous role in formation of the nervous system, I predicted that reduced levels of FoxP would impair courtship song and other types of complex locomotion in ways that parallel vocal and motor impairments seen in vertebrate FoxP2 studies. To test this I employed the UAS-GAL4 system with a FoxP specific UAS-RNAi to knockdown expression of FoxP in specific tissue types. A fter confirming successful knockdown we recorded several aspects of fly behavior, including courtship song, walking, and flight. We found deficits in all of these behaviors in adults, with males more strongly affected than females in these assays. A dditionally, I created a FoxP-GA L 4 line which when combined with a conditional temperature sensitive UAS-shibire ${ }^{\text {ts }}$ line to transiently disrupt neurotransmission in FoxP expressing neurons, we observed dramatic effects on motor coordination. Our results provide the first characterization of FoxP's behavioral importance in invertebrates and suggest an intriguing homology with the crucial human speech and language gene, FOX P2.

\subsection{M aterials and methods}

\section{Animals}

D rosophila melanogaster fruit flies were maintained at room temperature $\left(23-25^{\circ} \mathrm{C}\right)$ or in a $29^{\circ} \mathrm{C}$ incubator on standard yeast and glucose media. We reduced FoxP mRNA expression by crossing GA L4 driver lines with a UAS-RNAi construct specific to FoxP: UAS-F oxPIR (15732) from the V ienna D rosophila Stock Center (Dietzl et al. 2007). Two different GA L4 drivers were 


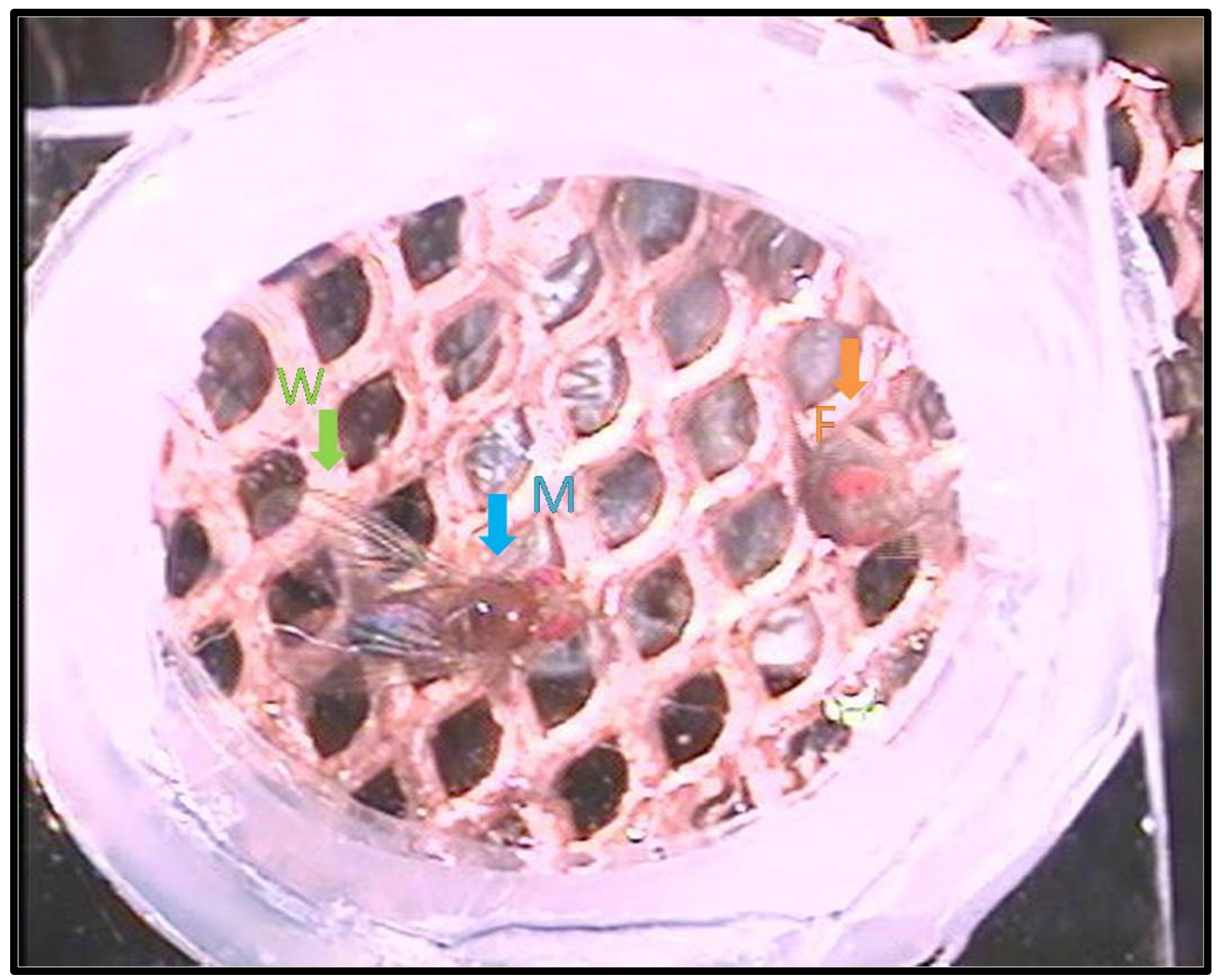

Figure 2. A male fly courting a female in a recording chamber. A $n$ individual male placed with a virgin female will court vigorously in the above chamber, while a microphone underneath records song and a camera above captures courtship behavior. The male (M) orients to the female $(\mathrm{F})$ and extends a vibrating wing $(\mathrm{W})$ to produce courtship song. 
crossed to the UAS-RNAi line, including the pan-neural elav-GAL4 (FBst0000458) and the ubiquitously expressed A ct5c-GA L 4 (FB st0003954). We also generated a GAL4 line driven by the putative FoxP promoter (FoxP-Gal4), which was crossed with the temperature sensitive UAS-Shit $^{\text {ts1 }}$ line (FBst0044222) to selectively disrupt FoxP neuron function. This line contains a temperature sensitive mutation in the shibire gene, which codes for a semi-dominant form of the endocytotic protein dynamin (Kitamoto 2001). This allowed for silencing of any neurons expressing this mutation when the fly was placed at an elevated temperature. When combined with our FoxP-GA L4, Shits ${ }^{\text {ts }}$ was only present in FoxP expressing cells.

RNAi efficacy

RNAi knockdown was assessed using RT-PCR. RNA was extracted from 30 adult fly heads using Trizol (Invitrogen, Carlsbad, CA, USA), and lug of RNA was reverse transcribed using Superscript III (Invitrogen, Carlsbad, CA, USA) according to the manufacturer's directions. FoxP CDNA was amplified by PCR using the primers 5'CCCATCCGACAAACAAATTC-3' and 5'-TCACATTCTCAACCCGCATA-3', Failsafe (Epicentre, Madison, WI, USA) with Buffer $\mathrm{D}$, and the following program: $2 \mathrm{~min}$ at $94^{\circ} \mathrm{C}$ for 1 cycle; $15 \mathrm{~s}$ at $94^{\circ} \mathrm{C}, 15 \mathrm{~s}$ at $47^{\circ} \mathrm{C}, 45 \mathrm{~s}$ at $72^{\circ} \mathrm{C}$ for 35 cycles; 5 min at $72^{\circ} \mathrm{C}$ for 1 cycle. The ribosomal marker Rp49 (Primers 5'-AAGATGACCATCCGCCCAGCA-3' and 5'CCCTTGAAGCGGCGACGC-3') was used as a control and all PCR products were separated by gel electrophoresis for identification.

Transgenic fly generation

For creation of the FoxP-GAL4 line, a 1.5kb fragment of genomic DNA upstream of the Foxp gene (5'-CCGGATCCTGTTTTTAAAACTGAAATTTATAATCATTACCATTG-3' and 
5'-CCGGTACCGCCTTAGGATGCATTTTGAAAC-3' containing the underlined BamHI and $\mathrm{K} \mathrm{pnl} \mathrm{restriction} \mathrm{sites,} \mathrm{respectively} \mathrm{for} \mathrm{each} \mathrm{primer)} \mathrm{was} \mathrm{used} \mathrm{as} \mathrm{the} \mathrm{putative} \mathrm{promoter} \mathrm{region} \mathrm{and}$ amplified by PCR using DyNAzyme EXT DNA polymerase (New England Biolabs, Ipswich, MA, USA), which was then cloned into the $\mathrm{pGaTB} G A L 4$ vector. The resulting construct of the FoxP promoter and GAL4 was subcloned into the $\mathrm{pC}$ aSpeR 2 P-element transformation vector. This construct was injected into embryos (B estgene, Chino Hills, CA, USA), and transformants were selected based upon CNS fluorescence expression when combined with a UAS-CD 8::GFP reporter line.

Behavior

For courtship assays, individual males were anesthetized with $\mathrm{CO}_{2}$ a few hours posteclosion and raised in isolation prior to testing. A ssays were performed 4-6 days later, to allow flies to mature and recover from $\mathrm{CO}_{2}$ anesthesia. For each recording experiment, a single virgin female (age 3-5 days) was paired with an individual male in a $10 \mathrm{~mm}$ diameter by $6 \mathrm{~mm}$ height plastic chamber with a copper mesh bottom (Rubinstein et al. 2010). Flies were gently aspirated into the chamber with a mouth pipette. The chamber was placed above a small microphone in a humidified box on a $25^{\circ} \mathrm{C}$ heat block to maintain suitable conditions to stimulate courtship. A microscope and attached camera recorded video of the courtship behavior to a computer. Recording started within 30 seconds of adding both flies to the chamber, and continued for 5 minutes or until successful copulation, whichever came first. Courtship behavior was recorded by hand from offline videos using Etholog (Ottoni 2000) by an observer blind to the male's genotype. Courtship index $(\mathrm{Cl})$ is the percentage of time a male courts, which was calculated as the time spent courting over the total time of the experiment. For knockdown female copulation 
latency $(C L)$ experiments, courtship was observed for 20 minutes or until successful copulation and the time to copulation was recorded.

Overall activity level was assayed using the DAM2 Drosophila Activity Monitor (Trikinetics, W altham, M A, USA ). Individual flies were placed in monitor tubes and the number of laser beam breaks per minute was automatically recorded for one hour for the RNAi knockdown experiment.

Flight ability was tested by placing groups of approximately 25 flies in a petri-dish on a heat block at a noxious temperature $\left(46^{\circ} \mathrm{C}\right)$ to induce a flight escape response. The assays were video recorded for offline data analysis. The petri-dish walls and ceiling were coated with fluon (Bioquip products, Rancho Dominguez, CA, USA) to prevent flies from crawling on these surfaces. A trial test using flies with clipped wings was performed to ensure that flies were unable to escape by jumping rather than flying. For the actual flight assay, groups of 20-25 intact flies were briefly anesthetized and placed in the dish one hour prior to testing to acclimate to their environment and recover from $\mathrm{CO}_{2}$ anesthesia. The dish was then placed on the heat block for 30 seconds before the lid was removed and the flies were allowed 30 seconds to escape from the noxious heat stimulus. The number of flies remaining at the end of the experiment was recorded from video analysis and used to calculate the proportion which escaped: Flight Index $(\mathrm{FI})=[\#$ flies at start $-\#$ flies remaining $] /[\#$ flies at start $]$.

The larval locomotion experiment used wandering third instar larvae which were placed individually on the center of a $15 \mathrm{~cm}$ petri dish with $0.07 \%$ agarose. This dish was placed on grid paper with $6 \mathrm{~mm}$ squares, and video recorded for three minutes. Offline analysis of the number of squares crossed per 30 seconds was used to calculate a mean rate of squares per time for each genotype and gender. 
For the acute shibire ${ }^{\text {ts }}$ experiments, observational recordings from several flies were performed with an individual fly from either a UAS-Shits1 control or a FoxP $>$ Shit ${ }^{\text {ts1 }}$ fly in a vial placed in an incubator at $37^{\circ} \mathrm{C}$. For quantification of these effects, flies were recorded in the DAM system in an incubator for a two hour series with 30 minutes of recording for each successive temperature with a range from room temperature $\left(23-25^{\circ} \mathrm{C}\right), 30^{\circ} \mathrm{C}, 35^{\circ} \mathrm{C}$, and room temperature again. Increasing the temperature by $5^{\circ} \mathrm{C}$ was observed to take 3 minutes for each of these intervals.

Statistics and analysis

Statistical analysis was performed using JMP 9 (SAS Institute Inc., Cary, NC, USA) using either student t-tests for data with two groups, or ANOVA with correction for multiple comparisons (Tukey HSD) for data with three or more groups. Courtship song analysis was performed using custom designed peak detection software. Audio signal was $100 \mathrm{~Hz}$ high pass filtered and denoised above $750 \mathrm{~Hz}$. Pulses below twice the noise level were ignored. The time point of a peak was defined as the maximum intensity of a pulse with a maximum width of 25 milliseconds. Computer identified pulses were reviewed by hand to confirm accuracy of the program as well as compared with video data to confirm wing extension behavior. A minimum of three consecutive pulses were required to be classified as a bout with a maximum of 125 milliseconds between pulses. For courtship index and larval locomotion, Etholog (Ottoni 2000) was used to manually record the percentage of time courting and the number of squares traversed over time, respectively. 


\subsection{Results}

\subsubsection{FoxP knockdown}

In order to assay phenotypic effects of reduced FoxP, we used RNA interference (RNAi) to produce knockdown of FoxP expression. RT-PCR confirmed reduced expression levels of FoxP mRNA from these flies. Expression of FoxP is noticeably reduced in adult heads using two types of GAL4 driver lines (the pan-neural elav-GAL4 and the ubiquitous Act5c-GAL4), al though faint expression remains, indicating a partial knockdown (Fig. 3).

\subsubsection{Courtship and song}

I hypothesized that reduction of FoxP levels would affect courtship song production in adult male flies, similar to sound production deficits seen in vertebrates. Courtship assays show a robust effect of FoxP knockdown on both courtship behavior and song. WT $(93 \%, n=29)$ and UAS-RNAi control males $(94 \% ; n=16)$ vigorously court females throughout the length of the assay, whereas only $65 \%$ of elav>RNAi males $(n=23)$ raised at room temperature exhibit any courtship behavior (Fig. 4A). Elav>RNAi males $(n=15)$ who do court and sing show a significant reduction in courtship index $(\mathrm{Cl})$, which is the proportion of time spent courting, as compared to both WT ( $n=27 ; P<0.0001)$ and UAS-RNAi ( $n=15 ; P=0.0003)$ controls (Fig. 4B). We also tested whether FoxP knockdown in females affects courtship behavior in normal males using courtship index (Fig. 5A) and copulation latency (Fig. 5B) assays, but observed no significant differences (UAS-RNAi $n=14$; elav>RNAi $n=13 ;$ Act5c $>$ RNAi $n=13 ; p>0.7$ for all group comparisons). 


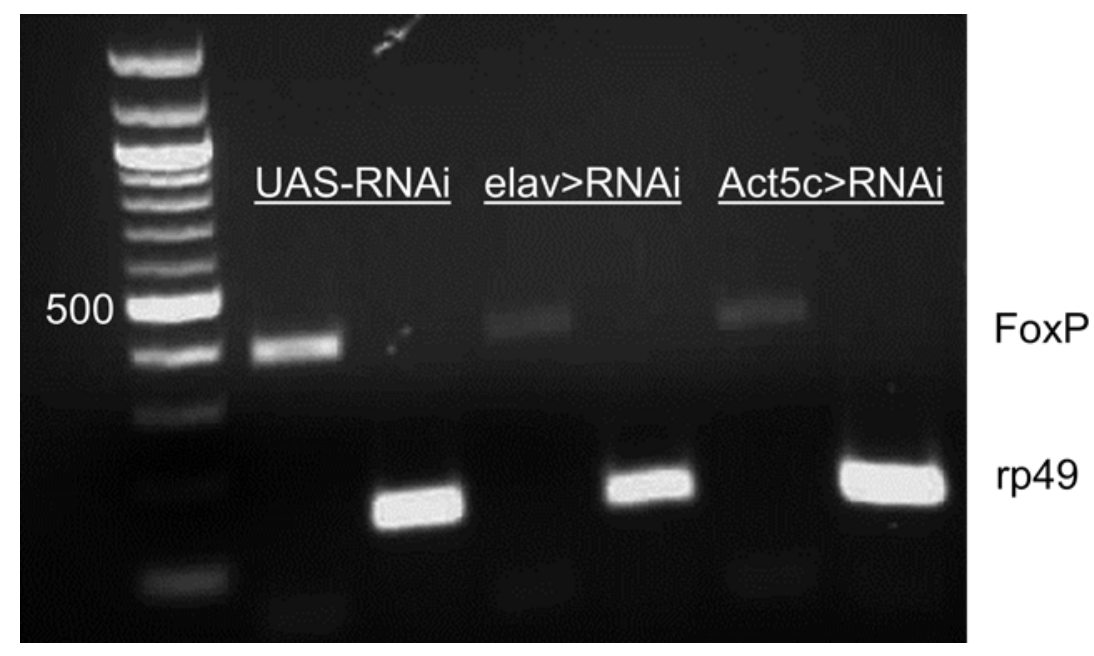

Figure 3. Confirming knockdown of FoxP expression. A. RT-PCR of FoxP indicates successful partial knockdown of FoxP RNA in adult fly head tissue. The UAS-RNAi control shows strong expression without knockdown. Elav>RNAi and Act5c>RNAi knockdown flies exhibit reduced FoxP RNA, although some faint expression remains. R p49 primers were used as an RT-PCR control. 
During the knockdown male courtship experiments, we concurrently recorded the male pulse song (Fig. 6A) and found that song structure is also altered in FoxP knockdown flies, with a faster and more variable inter-pulse-interval (IPI) (Fig. 6B; $P=0.0291$ ), longer pulse song bouts (Fig. 6C; $P=0.0291$ ), and a greatly reduced percentage of time spent singing (Fig. 6D; $P=0.0069$ ). We also created a stronger knockdown by raising flies at $29^{\circ} \mathrm{C}$, which increases the efficacy of the GAL 4 (B rand \& Perrimon 1993), but all elav>R NA i males failed to court.

\subsubsection{Locomotion and flight}

Based on subtle locomotion deficits observed in heterozygous FoxP2 mutant mice, we predicted that the effect of FoxP knockdown in flies might have a generalized effect on motor coordination in other behaviors. To test this, we performed both flight and walking assays using FoxP RNAi with the same GAL4 drivers as in the courtship assays. In the flight assay, FoxP knockdown flies were less likely to use flight to escape from a heated dish after removal of the lid (Fig. 7A; $P<0.0001)$. This effect was stronger in males, with both elav>R NAi males ( $n=12$; $P<0.0001)$ and $A c t 5 c>R N A i$ males $(n=4 ; P<0.0001)$ significantly different compared to UASRNAi control males $(n=12)$. Whereas, Act5c>R NAi females showed a less significant decrease in flight escape ( $n=11 ; P=0.01112$ ) and elav $>$ R N A i females were not significantly different from UAS-RNA i control females ( $n=11 ; P=0.9840)$. U pon careful observation, knockdown flies that

did not escape were running rapidly in the bottom of the dish, indicating that the reduced flight is unlikely due to impaired heat sensitivity. 

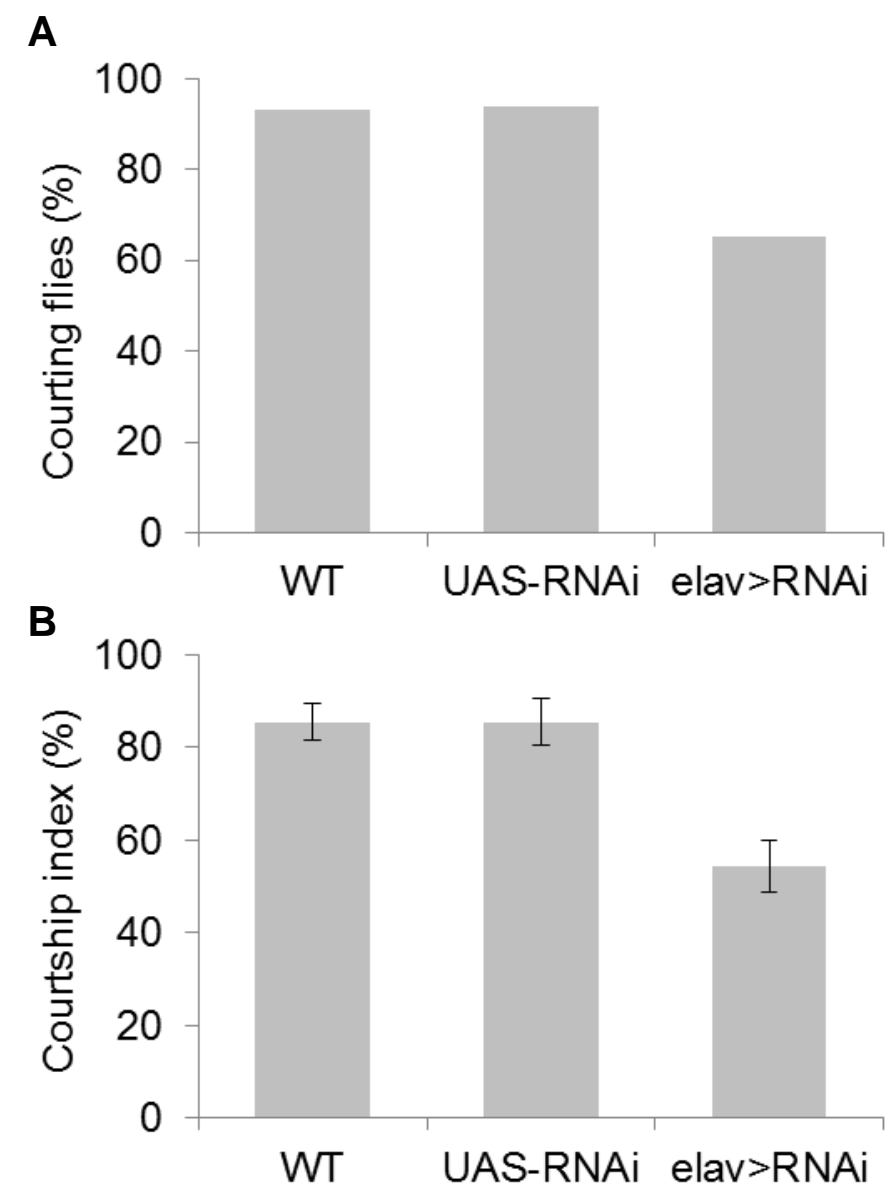

Figure. 4. Effects of FoxP knockdown on male courtship behavior. RNAi mediated knockdown of FoxP reduces courtship in male flies. ${ }^{* * P}<0.005$. A. Fewer elav $>$ RNAi knockdown males ( $n=23$ ) court or sing compared to controls (UAS-RNAi $n=16$; WT $n=29$ ) (mean \pm SEM ). B. K nockdown males that do sing $(n=15)$ spend significantly less time courting than controls (UAS-RNA i $n=15$; W T $n=27$ ). 

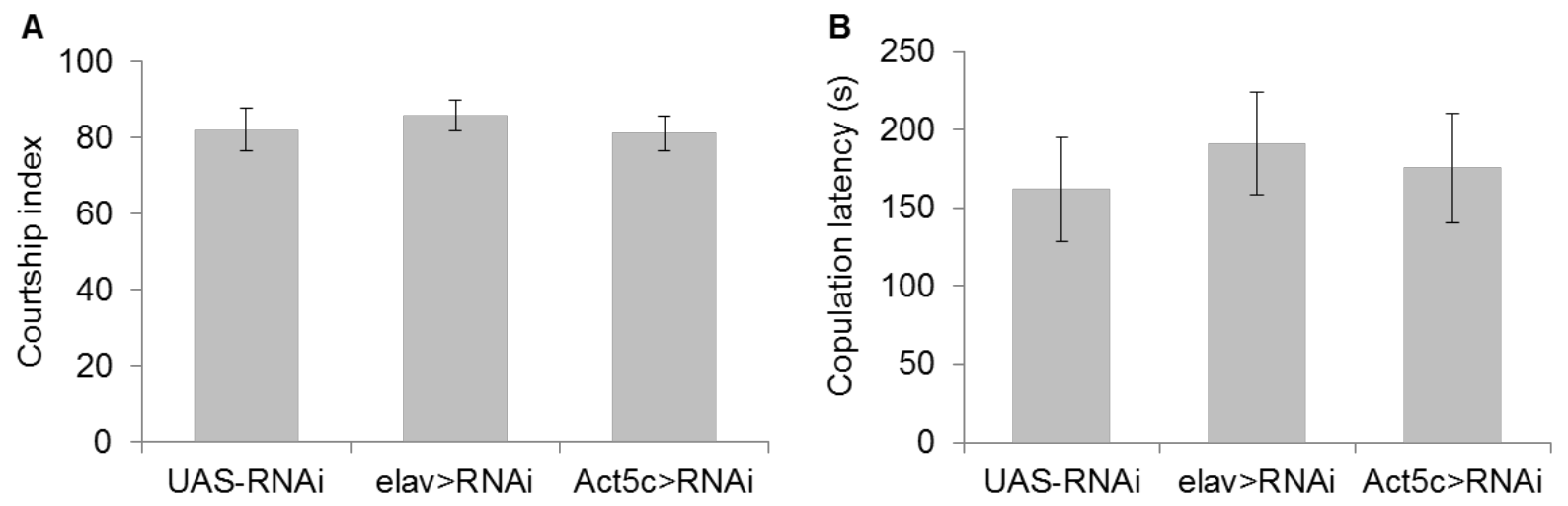

Figure 5. Effects of female F oxP knockdown on male courtship behavior. RNAi knockdown females do not induce a significant courtship behavior difference in UAS-RNA i control males as measured by $\mathbf{A}$. courtship index (UAS-RNA i $n=14$; elav>RNA i $n=13$; $A$ ct5c $>$ RNA i $n=13$ ) or $\mathbf{B}$. copulation latency (UAS-RNA i $n=14$; elav>RNA i $n=12$; $A$ ct5c $>$ RNA i $n=13$ ). Mean $\pm S E M$ for each group. 

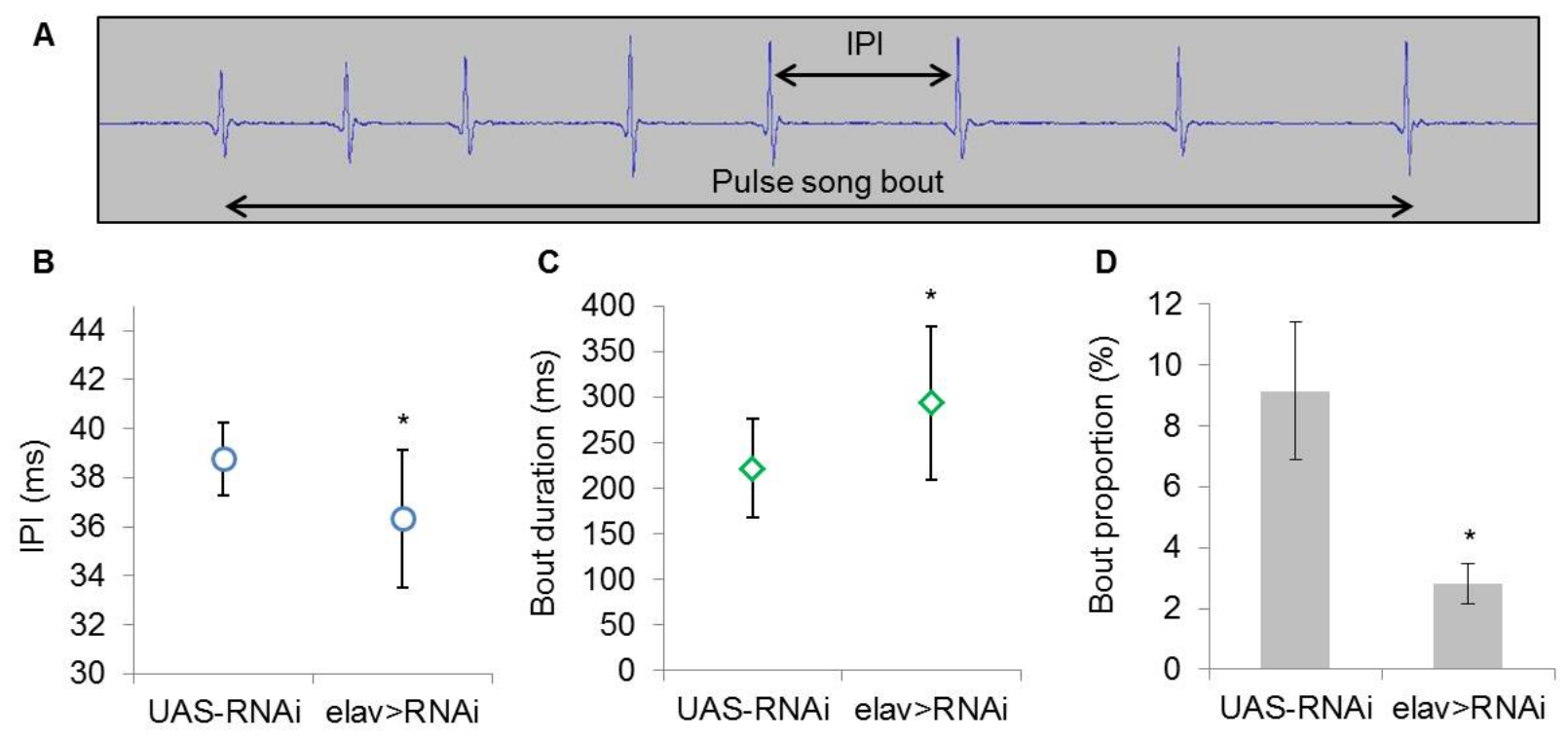

Figure 6. Effects of FoxP knockdown on courtship song. Pulse song structure is altered in FoxP knockdown males. M ean \pm S.D. for $B$ and $C$, and S.E.M for $D$. $* P<0.05$. A. Oscillogram example of a UAS-RNAi pulse song bout with the inter-pulse interval (IPI) and bout duration labeled. B. IPI is reduced and more variable in knockdown males $(n=12)$ compared to controls $(n=9)$. C. A verage song bout duration is increased in knockdown males. D. Knockdown males show a significant decrease in proportion of time spent singing. 
Walking ability in adults was measured using general activity level as assayed by number of beam breaks per minute in a D rosophila automated monitoring system (DA M S, Trikinetics). Both male and female RNA i knockdown flies for either driver type were significantly less active compared to controls over a one hour DAM S experiment (Fig. 7B; $\mathrm{P}<0.001$ ). elav $>$ RNA i males $(n=30 ; P<0.0001)$ and Act5c $>$ RNAi males $(n=32 ; P<0.0001)$ were significantly less active than UAS-RNAi control males ( $n=33$ ). Once again, this effect was larger in males, but unlike in the flight assay, both elav>RNAi females ( $n=33 ; P=0.0115$ ) and Act5c>RNAi females ( $n=33$; $P<0.0001)$ were significantly less active than UAS-RNAi control females $(n=33)$.

FoxP knockdown larvae had a large increase in activity as measured by the rate of boxes crossed per time (Fig. 7C). These flies exhibited less frequent turning behavior and often reached the edge of the plate before the controls. No obvious difference between genders was observed.

In order to more closely examine the behavioral role of FoxP expressing cells, we created a FoxP specific GAL4 line. When combined with the temperature sensitive dynamin mutant UAS-Shits ${ }^{\text {ts1 }}$ line to temporarily impair synaptic transmission in adult FoxP neurons, we see dramatically disturbed movement during heating (Fig. 8). Observationally, within a few minutes at elevated temperatures the FoxP $>\mathrm{Shi}^{\text {ts }}$ flies drop to the floor of the vial and begin to display extremely uncoordinated and abnormal walking behavior such as jerky sideways and backwards walking. They are unable to climb the walls of the vial, and they produce spastic movements along the bottom of the vial. Eventually they slow and appear to walk less and often stop moving, but do not paralyze. It was also noted that these flies appear to have an impaired righting reflex, with extended time needed to right themselves when flipped onto their backs.

Using the DAM system to quantify the change in coordination in these flies, we see a dramatic difference in activity in both males and females at $35^{\circ} \mathrm{C}$ as compared to room 
temperature (Fig. 9). Initially all groups show similar low activity levels at room temperature, which then increase at higher temperatures. At $30^{\circ} \mathrm{C}$ all groups have slightly increased activity relative to room temperature. At $35^{\circ} \mathrm{C}$ the UAS-Shits ${ }^{\text {tsl }}$ controls greatly increase activity, but the $\mathrm{F}$ oxP $>\mathrm{Shi}^{\text {ts1 }}$ flies do not show the same dramatic increase in activity. All groups return to low activity levels after removal from heat. 

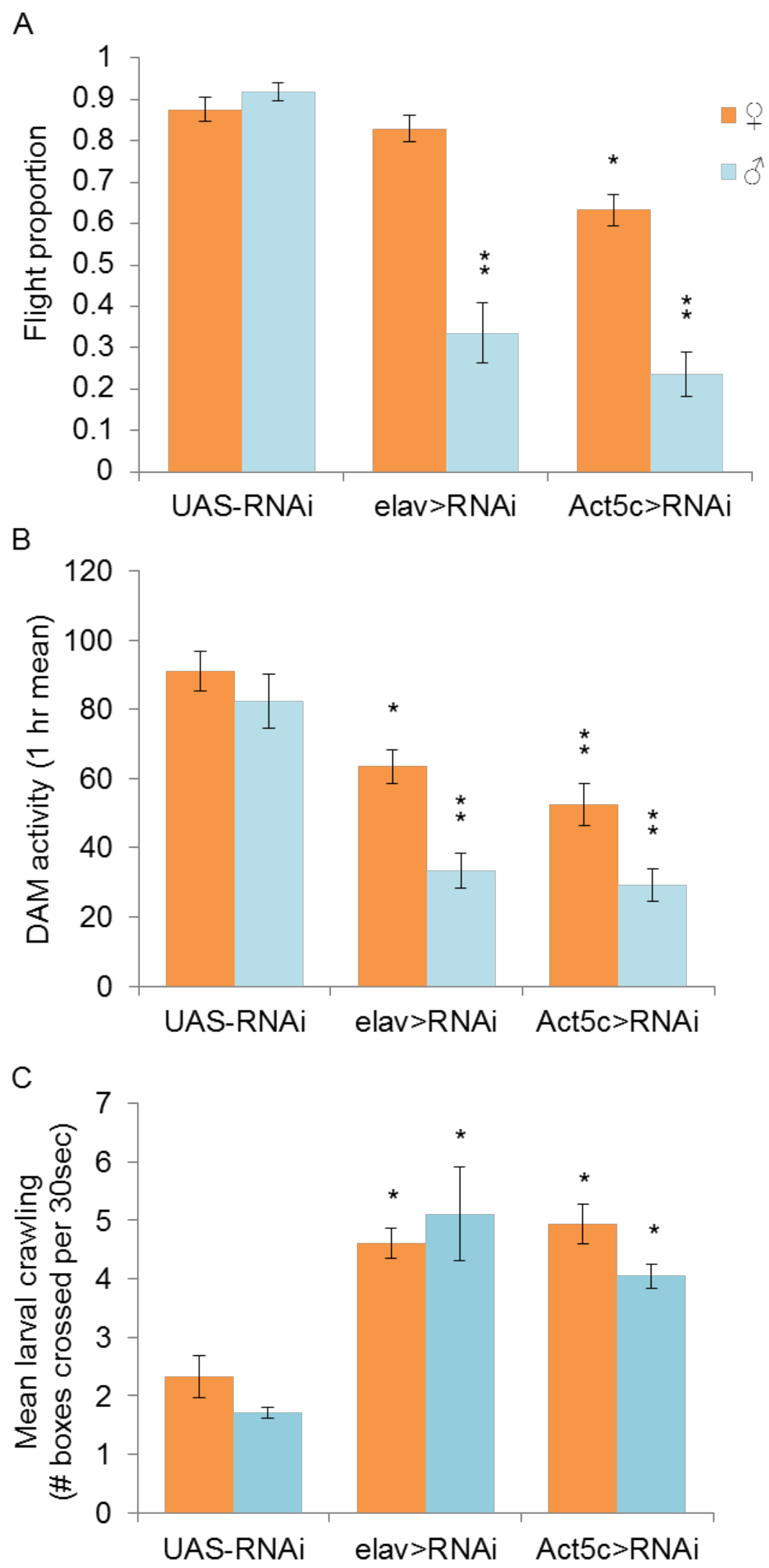
Figure 7. Effects of FoxP knockdown on locomotion and flight. FoxP knockdown impairs locomotion and flight, with a much greater effect in males (mean \pm SEM). Symbols indicate significant difference from gender matched control. $* P<0.05, * * P<0.005$. A. A lower percentage of FoxP knockdown flies (elav>RNA i $n=27 ; A c t 5 c>R N A i n=15$ ) escape in a heated flight assay as compared to controls (UAS-RNAi $n=23$ ). B. Locomotion activity is also decreased in FoxP knockdown flies ( $n=11$ each group). C. FoxP knockdown in late stage larvae indicates increased locomotion for both groups compared to controls, with no significant difference between genders. 


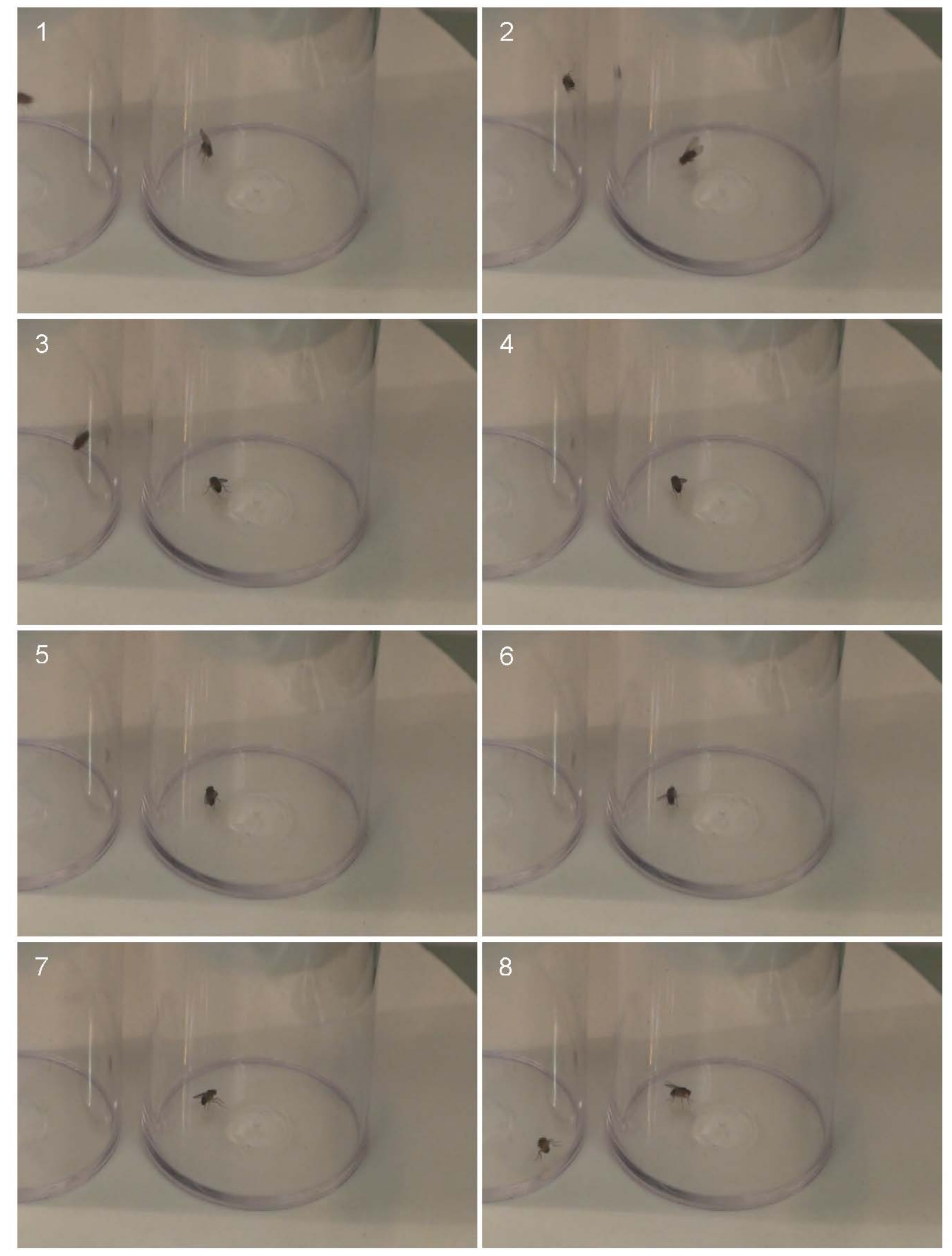


Figure 8. Walking behavior after disrupting neurotransmission in FoxP expressing cells. Time-lapsed images show that at $35^{\circ} \mathrm{C}, \mathrm{FoxP}>$ shibire ${ }^{\text {ts }}$ flies (right) are no longer able to crawl on the side of the vial, and they exhibit spastic uncoordinated movement along the bottom of the vial, resulting in little forward progress. UAS-shibire ${ }^{\text {ts }}$ controls (left) continue to run rapidly on all surfaces of the vial, occasionally disappearing from view. Individual images are still frames captured every one second from eight seconds of video recorded after two minutes in the incubator. 


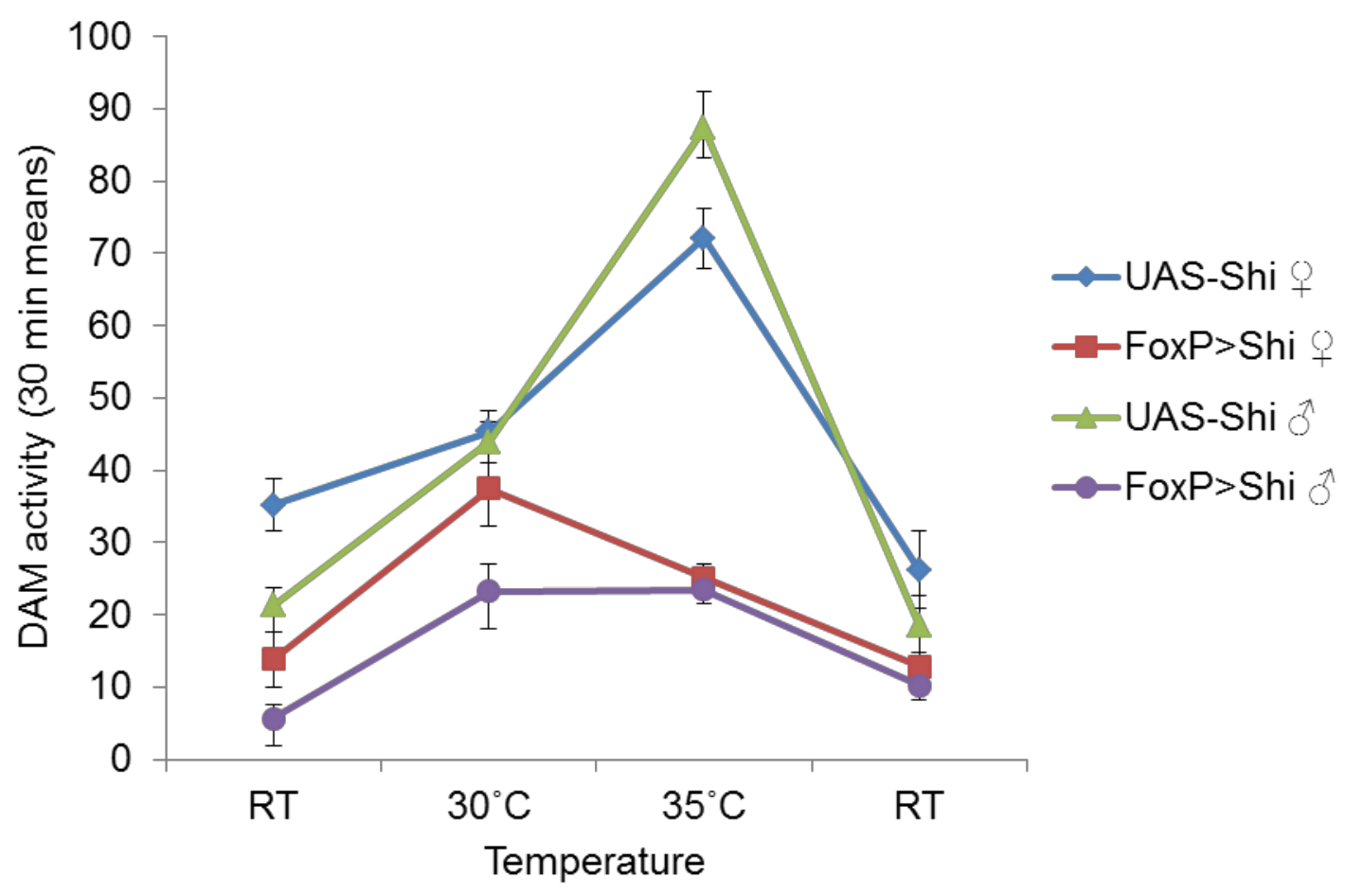

Figure 9. Q uantification of average activity level after acute disruption of FoxP expressing neurons using a temperature sensitive UAS-shibire ${ }^{\text {ts }}$ line in adult flies. The Drosophila activity monitor (DAM) was used to monitor average activity level over 30 minutes for each temperature (mean $\pm \mathrm{SEM}$ ). R oom temperature $(\mathrm{RT})$ varied between $23-25^{\circ} \mathrm{C}$. Unlike controls, activity level for FoxP $>\mathrm{Shi}^{\text {ts }}$ flies decreases dramatically at $35^{\circ} \mathrm{C}$ for both sexes. 


\subsection{Discussion}

FoxP2, a crucial speech and language gene in humans, affects sound production and motor skills in other organisms and is thought to be involved in synaptic plasticity and development in brain regions known to be important for fine motor control (Fisher \& Scharff 2009). Despite several years of study, the mechanism of action of FoxP2 in vertebrate nervous systems is not well understood. The fly homolog FoxP is similar in sequence and expression, but its function has yet to be addressed. Our research indicates that Drosophila FoxP also plays a role in motor coordination in the CNS.

Successful partial knockdown of FoxP using RNAi in flies mimics heterozygous mutations in humans and mice, as well as RNAi used in zebra finch. In our study, no gross developmental abnormalities are apparent in knockdown flies. These flies, as in vertebrates with partial FoxP2 deficiency, show specific motor deficits without any obvious external morphological aberrations. With the stronger $29^{\circ} \mathrm{C}$ knockdown of $\mathrm{Act} 5 \mathrm{C}>\mathrm{RNAi}$ we do observe pupal lethality in males - this is consistent with results in homozygous null mutant FoxP2-mice, which have severe developmental defects and die around 3 weeks of age (Groszer et al. 2008; Fujita et al. 2008; Shu et al. 2005). FoxP may be playing an essential role in neural development and it is possible that FoxP plays distinct roles in various tissues at different time points in development, as this is a common trait of transcription factors. The crucial timing of FoxP action will be addressed in the following chapter.

Given the role of FoxP2 in human speech, zebra finch song learning, and possibly mouse pup cries, we predicted that FoxP would also play a role in sound production in insects. Our results indicate that this is indeed the case, although given the other motor impairments, the effects on courtship and song are likely related to motor coordination impairments, rather than a 
specific disruption of the song circuitry. This is supported by previously reported results in mice, where effects on pup cries were one of many deficits, including motor skill learning and synaptic plasticity (G roszer et al. 2008; K urt et al. 2012; French et al. 2012). Partially reduced FoxP does not completely eliminate song production in the majority of flies, but does change song structure, which also supports the idea of disrupted fine motor control rather than a more general inability to produce or maintain movement. Strongly convincing support for this idea comes from the dramatic effects on walking using the $\mathrm{FoxP}>\mathrm{Shi}^{\mathrm{is} 1}$ flies. These results demonstrate that FoxP expressing neurons are crucial for maintaining proper motor coordination, but without abolishing movement completely. This result is especially notable for being an acute disruption of FoxP neurons, rather than a chronic disruption throughout development using the RNAi knockdown. This reduces the likelihood of compensatory mechanisms and off target RNA i effects.

Unexpectedly, we observed that impairment in the majority of motor tasks was more severe in males than females. In both the walking and flight assays, males were strongly affected, with only a slight but significant impairment in Act5c>R NA i females. Elav>R NA i females exhibit no significant difference in the flight assay, and only a slight decrease in walking activity level. This is likely because the neural elav-GAL4 is a weaker driver than Act5c-GAL4 (Schroeder \& Jackson 2002). We cannot rule out the possibility of a greater sensitivity to the RNAi in males ( $\mathrm{Ni}$ et al. 2008), but it may be that FoxP itself plays different roles in the two sexes. While the majority of vertebrate FoxP2 studies have not addressed sex specific behavioral differences, a few studies have found differences in expression level of FoxP2 in rodents and humans, as well as a behavioral difference in pup calls between the sexes (Hamson et al. 2009; B owers et al. 2013). It is al ready known that normal locomotion in flies is sexually dimorphic (M artin 2004), which is not unexpected given their many non-overlapping behaviors such as male courtship and 
fighting versus female egg laying. These sex differences raise the intriguing question of whether any gender differences might be observed in future FoxP2 studies in vertebrates.

This work establishes the behavioral effects of FoxP knockdown in an invertebrate with functional parallels to the vertebrate FoxP2. Further work addressing the mechanisms of action of this gene in the nervous system may contribute to understanding elements of Drosophila brain development, as well as provide valuable insight into the evolutionarily conserved functions of the FoxP homologs across invertebrates and vertebrates. In the following chapter I will address the expression pattern and crucial timing of action of FoxP in the fly CNS. 


\section{REFERENCES}

Bishop, D.V.M., 2006. What Causes Specific Language Impairment in Children? Current directions in psychological science/: a journal of the American Psychological Society, 15(5), pp.217-221.

Bowers, J.M . et al., 2013. Foxp2 mediates sex differences in ultrasonic vocalization by rat pups and directs order of maternal retrieval. The Journal of neuroscience/: the official journal of the Society for Neuroscience, 33(8), pp.3276-83.

Brand, A.H. \& Perrimon, N., 1993. Targeted gene expression as a means of altering cell fates and generating dominant phenotypes. D evelopment (Cambridge, England), 118(2), pp.40115.

Chintapalli, V.R., Wang, J. \& Dow, J.A.T., 2007. U sing FlyA tlas to identify better Drosophila melanogaster models of human disease. Nature genetics, 39(6), pp.715-20.

Dietzl, G. et al., 2007. A genome-wide transgenic RNA i library for conditional gene inactivation in Drosophila. Nature, 448(7150), pp.151-6.

Enard, W. et al., 2002. M olecular evolution of FOXP2, a gene involved in speech and language. Nature, 418(6900), pp.869-72.

Ferland, R.J. et al., 2003. Characterization of Foxp2 and Foxp1 mRNA and protein in the developing and mature brain. The J ournal of comparative neurology, 460(2), pp.266-79.

Fisher, S.E. \& Scharff, C., 2009. FOXP2 as a molecular window into speech and language. Trends in genetics/: TIG , 25(4), pp.166-77.

French, C.A. et al., 2012. An aetiological Foxp2 mutation causes aberrant striatal activity and alters plasticity during skill learning. M olecular psychiatry, 17(11), pp.1077-85.

Fujita, E. et al., 2008. Ultrasonic vocalization impairment of Foxp2 (R552H) knockin mice related to speech-language disorder and abnormality of Purkinje cells. Proceedings of the National Academy of Sciences of the U nited States of America, 105(8), pp.3117-22.

Gaub, S. et al., 2010. The structure of innate vocalizations in Foxp2-deficient mouse pups. Genes, brain, and behavior, 9(4), pp.390-401.

Groszer, M. et al., 2008. Impaired synaptic plasticity and motor learning in mice with a point mutation implicated in human speech deficits. Current biology/: CB , 18(5), pp.354-62.

Haesler, S. et al., 2004. FoxP2 expression in avian vocal learners and non-learners. The J ournal of neuroscience/: the official journal of the Society for Neuroscience, 24(13), pp.3164-75. 
Haesler, S. et al., 2007. Incomplete and inaccurate vocal imitation after knockdown of FoxP2 in songbird basal ganglia nucleus A rea X. PL oS biology, 5(12), p.e321.

Hamson, D.K. et al., 2009. A nalysis of Foxp2 expression in the cerebellum reveals a possible sex difference. Neuroreport, 20(6), pp.611-6.

K itamoto, T., 2001. Conditional modification of behavior in Drosophila by targeted expression of a temperature-sensitive shibire allele in defined neurons. J ournal of neurobiology, 47(2), pp.81-92.

Kurt, S., Fisher, S.E. \& Ehret, G., 2012. Foxp2 mutations impair auditory-motor association learning. PloS one, 7(3), p.e33130.

Lai, C.S. et al., 2001. A forkhead-domain gene is mutated in a severe speech and language disorder. Nature, 413(6855), pp.519-23.

Lee, H. \& Frasch, M., 2004. Survey of forkhead domain encoding genes in the Drosophila genome: Classification and embryonic expression patterns. Developmental dynamics/: an official publication of the American Association of Anatomists, 229(2), pp.357-66.

MacDermot, K.D. et al., 2005. Identification of FOXP2 truncation as a novel cause of developmental speech and language deficits. American journal of human genetics, 76(6), pp.1074-80.

M artin, J.-R., 2004. A portrait of locomotor behaviour in Drosophila determined by a videotracking paradigm. Behavioural processes, 67(2), pp.207-19.

M arygold, S.J. et al., 2013. FlyB ase: improvements to the bibliography. Nucleic acids research, 41(Database issue), pp.D 751-7.

$\mathrm{Ni}$, J. et al., 2008. Vector and parameters for targeted transgenic R NA interference in Drosophila melanogaster. Nature methods, 5(1), pp.49-51.

Ottoni, E.B., 2000. EthoL og 2.2: a tool for the transcription and timing of behavior observation sessions. Behavior research methods, instruments, \& computers/: a journal of the Psychonomic Society, Inc, 32(3), pp.446-9.

Rubinstein, C.D., Rivlin, P.K . \& Hoy, R.R., 2010. Genetic feminization of the thoracic nervous system disrupts courtship song in male Drosophila melanogaster. J ournal of neurogenetics, 24(4), pp.234-45.

Santos, M .E. et al., 2011. A Iternative splicing and gene duplication in the evolution of the FoxP gene subfamily. Molecular biology and evolution, 28(1), pp.237-47. 
Schroeder, A.J. \& Jackson, F.R., 2002. Phenotypic and molecular characterization of GA L4/UA S-mediated LARK expression. Genesis (New York, N.Y./: 2000), 34(1-2), pp.914.

Shu, W. et al., 2005. A ltered ultrasonic vocalization in mice with a disruption in the Foxp2 gene. Proceedings of the National Academy of Sciences of the U nited States of America, 102(27), pp.9643-8.

Tomblin, J.B. et al., 1997. Prevalence of specific language impairment in kindergarten children. J ournal of speech, language, and hearing research/: J SL HR, 40(6), pp.1245-60.

Zhao, Y unjing et al., 2010. A ssociation between FOXP2 gene and speech sound disorder in Chinese population. Psychiatry and clinical neurosciences, 64(5), pp.565-73. 


\section{CHAPTER 3}

\section{SPATIAL AND TEMPORAL ANALYSIS OF FOXPIN THE NERVOUS SY STEM}

\subsection{Abstract}

The vertebrate transcription factor FoxP2 is mutated in a multigenerational human family with a severe speech and language disorder. FoxP2 is known to be strongly expressed in brain regions important for motor coordination, such as the basal ganglia and the cerebellum but little is known about FoxP2's precise role in the nervous system. The recent discovery of the well-conserved Drosophila melanogaster homolog, FoxP, provides an opportunity to study the role of this crucial gene in an invertebrate model with a diverse suite of molecular tools. Utilizing a GFP reporter to visualize FoxP in the fly brain reveals expression in relatively few neurons in distributed clusters within the larval and adult CNS, including distinct labeling of the adult protocerebral bridge. This structure is a region of the insect central complex known to be important for motor coordination in insects and thought to be homologous to areas of the vertebrate basal ganglia. A temperature dependent disruption of FoxP neuronal activity during pupation demonstrates that FoxP is essential for successful eclosion behavior, suggesting a possible role in the dramatic changes in the nervous system during the transition from larva to adult. Our results suggest a role for FoxP in construction of specific brain regions important for fine motor coordination, supporting a deep homology in brain development between insects and humans. 


\subsection{Introduction}

Impairments in speech and language are usually evident early in development with delayed onset or unusual speech production in young children (Tomblin et al. 1997). The critical nature of proper speech production in human society creates a great need for prevention and treatment of these disorders. Some brain regions are already known to play crucial roles in speech production and language comprehension, but contribute only a small piece to understanding the complexity of language development. The discovery of the human KE family with severe language deficits due to a mutation in the transcription factor FOX P2 presents an opportunity to investigate language related brain regions in more detail (Lai et al. 2001).

Examination of affected KE family members' brains showed several abnormalities in both form and function. Even before the discovery of FOXP2, magnetic resonance imaging (M RI) scans identified structural differences relative to controls, with abnormal amounts of gray matter in several motor areas, such as parts of the basal ganglia, sensorimotor cortex, and cerebellum (V argha-K hadem et al. 1998; W atkins \& V argha-K hadem 2002). Language tasks performed while using positron emission tomography (PET) (V argha-K hadem et al. 1998) and functional magnetic resonance imaging (fM RI)(Liégeois et al. 2003) also highlighted differences in brain activity, including more widespread activation in affected family members versus controls. Striatal structures stood out as different in both size and activity level (V argha-K hadem et al. 1998; Watkins \& V argha-K hadem 2002; Liégeois et al. 2003). These studies directly compared the affected KE family members to controls in order to identify differences associated with the mutation, but these results were only able to identify changes late in development. This information may not be representative of early direct effects of FOXP2, but instead reflect the result of compensatory mechanisms or plasticity in language learning possibly facilitated by 
speech training during childhood. It is difficult to interpret the connection between this data with FOX P2's direct contribution to changes in these regions.

Complementing the structural and functional imaging, molecular techniques revealed FoxP2 expression in early development. When FoxP2 in mouse and human fetal brain were compared, both organisms show expression in similar brain regions at early time points in development. Particularly strong expression in motor areas such as the striatum and cerebellum is consistent with the morphological and activity changes in the KE family (Ferland et al. 2003; Lai et al. 2003). In several other vertebrate species similar FoxP2 expression patterns in early neural development are evident, with expression continuing into adulthood in several structures (Scharff \& Petri 2011).

The importance of this gene in neural development was further emphasized in studies which manipulated FoxP2 expression in model organisms. In juvenile zebra finches, FoxP2 knockdown during the song learning period results in incorrectly formed adult songs. This was accomplished by injection of FoxP2 RNAi specifically into A rea X (Haesler et al. 2007), which exhibits features similar to the mammalian striatum (Carrillo \& Doupe 2004). Further studies with A rea $\mathrm{X}$ have found FoxP2 target gene expression during song learning (Graham \& Fisher 2012). M ouse models with reduced FoxP2 expression also exhibited abnormal synaptic plasticity in the striatum and cerebellum (Fisher \& Scharff 2009; French et al. 2012). Whereas, mice altered to express the humanized version of FoxP2 showed opposite effects on synaptic plasticity in the striatum (Enard et al. 2009; R eimers-Kipping et al. 2010). Thus several studies in multiple organisms have emphasized the striatum as an important area of FoxP2 action (Graham \& Fisher 2012). 
The fruit fly D rosophila melanogaster FoxP is highly conserved with FoxP2 and it is also expressed early in the nervous system (Lee \& Frasch 2004), with continued expression throughout development. RNA-seq experiments indicate that FoxP expression is relatively low during most life stages, but higher in early to mid pupal stages and peaks at the mid-pupal stage P8 (Graveley et al. 2011). Given the variety of well-established molecular tools available in this organism to look at brain expression and function at different time-points, flies are an attractive system to explore this well-conserved gene. Some of these tools include specific antibodies or libraries of transgenic lines, which are useful for both visualization and manipulation of specific genes.

As a first step towards understanding the specific role of FoxP in the nervous system, we sought to define the spatial and temporal pattern of FoxP expression with the aim of linking this knowledge back to developmental and behavioral effects. Based on known expression patterns in FoxP2, we predicted that insect FoxP would be expressed in discrete brain regions important for motor coordination. We also expected to see expression differences between the genders, given the sex specific behavioral effects from our previous work. Our predictions also pinpointed pupation as a likely time of FoxP action in the nervous system, given that this is the moment of peak expression and a period of complete reconstruction of the nervous system. In order to explore these questions, we generated two molecular tools: a FoxP antibody and a FoxP-GA L4 line which, when combined with two different UAS-GFP lines, allowed for visualization of the expression pattern of FoxP in the larval and adult CNS. This revealed that FoxP is limited to relatively small subsets of neurons in the brain and ventral ganglion, which appear in several distinct clusters throughout. Particularly strong expression was evident in the protocerebral bridge, part of the central complex, which is thought to be involved in sensory-motor integration 
(Strauss 2002), and has been compared to the vertebrate basal ganglia (Strausfeld \& Hirth 2013), although no gender based difference was observed. When we used our FoxP-Gal4 with a conditional temperature sensitive UAS-shibire line to transiently disrupt neurotransmission in pupae we observed dramatic effects on eclosion behavior. A nimals failed to emerge from the pupal case, indicating a crucial role of FoxP+ neurons in this behavior.

\subsection{Materials and methods}

Animals

D rosophila melanogaster fruit flies were maintained at room temperature $\left(23-25^{\circ} \mathrm{C}\right)$ or in a $30^{\circ} \mathrm{C}$ incubator in vials with standard yeast and glucose media. We reduced FoxP mRNA expression by crossing the ubiquitously expressed Act5c-GA L 4 (FBst0003954) driver line with a UAS-R N A i construct specific to FoxP: UAS-F oxP IR (15732) from the Vienna D rosophila Stock Center (Dietzl et al. 2007). W e al so generated a GA L 4 line driven by the putative FoxP promoter (FoxP-Gal4), and recombined it with UAS-CD8::GFP (FBst0005130) and UAS-nls::GFP (FBst0004776) for visualizing the pattern of FoxP protein expression in the CNS. The FoxP-

Gal4 was also recombined with the temperature sensitive UAS-Shits1 line (FBst0044222) to selectively disrupt FoxP neuron function.

Antibody production

The polyclonal FoxP antibody was raised against a M altose Binding Protein-FoxP fusion. The fusion was generated by inserting a 462bp (154aa) PCR product from the FoxP coding region into the pMAL-c5X vector ( $\mathrm{New}$ England Biolabs, Ipswich, MA, USA). The fusion protein was injected into a guinea pig to produce the FoxP antibody (Pocono Rabbit Farm, 
Canadensis, PA, USA). The resulting antiserum was affinity purified as described in Risinger et al. 1997. The region used is not conserved with vertebrate $\mathrm{FoxP2}$, but is common to both $\mathrm{A}$ and $B$ isoforms of FoxP. Primers used were 5'-ATGCATCGGATACATGACGACGAGTATTC-3' and 5'-GAGTTCGCCATGCGGAAGTACTAT-3'.

Immunohistochemistry

For antibody staining, 3rd instar larva were dissected and fixed in 4\% paraformaldehyde for 30 minutes and processed as in Loveall and Deitcher 2010. A dults brains were dissected and fixed in 4\% paraformaldehyde for 20 minutes and processed as above. The following primary antibodies were used: the polyclonal guinea pig anti-FoxP (1:250) raised to the FoxP-MBP fusion protein, Rabbit anti-GFP (1:4000; Invitrogen), neuronal marker mouse anti-elav (1:400; Developmental Studies Hybridoma Bank), and the glial marker mouse anti- repo (1:30; Developmental Studies Hybridoma B ank). Secondary fluorescent antibodies used were goat antirabbit green A lexa 488 (1:2000; M olecular probes, Eugene, OR), donkey anti-guinea pig Cy3 red (1:1000; Jackson ImmunoResearch, Westgrove, PA, USA), and donkey anti-mouse Cy3 red (1:1000; Jackson ImmunoR esearch, W estgrove, PA, USA ). Fluorescence images were acquired using a Leica SP5 Confocal microscope (Cornell Imaging Facility) with a 20x objective.

\section{Eclosion timing}

Earlier behavioral experiments which attempted to use a stronger knockdown by placing Act $5 \mathrm{c}>\mathrm{RNAi}$ at $29^{\circ} \mathrm{C}$ resulted in a complete lack of eclosion behavior in males. Subsequently we examined eclosion in FoxP $>\mathrm{Shi}^{\text {its }}$ flies raised at $30^{\circ} \mathrm{C}$ to disrupt neurotransmission in FoxP cells at the pupal stage. For this experiment, wandering third instar larvae were transferred to fresh 
food vials (ten per vial) and kept at either room temperature $\left(23-25^{\circ} \mathrm{C}\right)$ or placed in a $30^{\circ} \mathrm{C}$ incubator for several days. The number of empty pupal cases were recorded and an eclosion percentage was calculated by dividing the number eclosed over the number which pupated. Larvae were selected randomly and not inspected for gender.

Statistics and analysis

Statistical analysis was performed using JMP 9 (SAS Institute Inc., Cary, NC, USA) using student t-tests. ImageJ (National Institutes of Health, Bethesda, MD, USA) was used for FoxP>nls::GFP cell counting.

\subsection{Results}

\subsubsection{Visualizing F oxP expression pattern}

In order to examine the expression pattern of FoxP in the CNS we used an antibody specific to FoxP and a FoxP-GAL4 line which we combined with the membrane bound UASCD8::GFP or the nuclear localized UAS-nls::GFP reporter lines to produce GFP specifically in FoxP expressing cells. Using the FoxP antibody on larval brains, we visually confirmed successful knockdown, with $A c t 5 c>R N A i$ flies showing greatly reduced FoxP expression as compared to controls (Fig. 1). Strongly overlapping expression of the pattern from these two independently created tools indicates a reliable FoxP staining pattern in larvae brain (Fig. 2).

FoxP appears to be limited to a relatively small number of cells in the larval and adult brain (Fig. 3). FoxP-GA L 4>CD 8::GFP expression in adult brain strongly labeled the distinct "handlebar" 


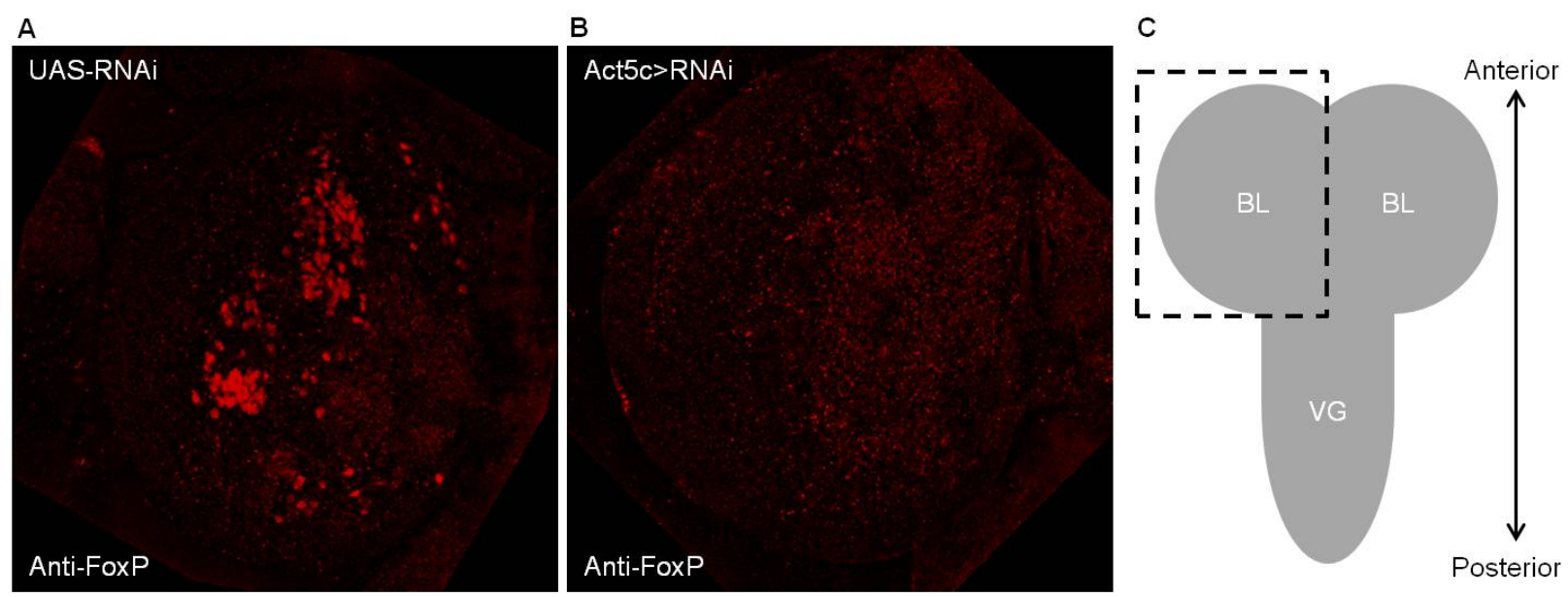

Figure 1. FoxP antibody staining in larva brain. A. Dissected larva brains stained with a FoxP antibody show distributed clusters of cells within an individual brain lobe. B. Larva with FoxP RNA i knockdown do not show expression with the antibody, confirming successful knockdown. C. Schematic of larva brain with dotted-line box indicating the region shown in $A$ and $B$ in two different brains. BL indicates the brain lobes and VG is the ventral ganglion. 

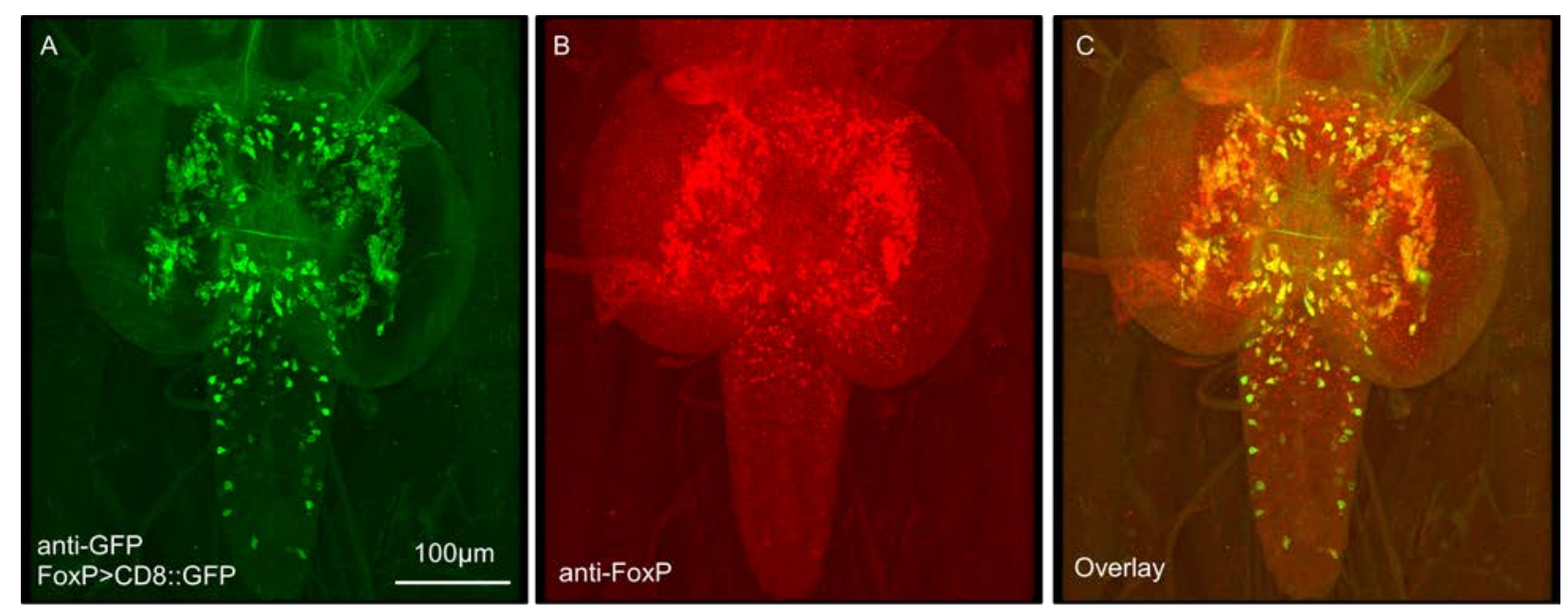

Figure 2. Overlap of FoxP antibody staining and FoxP-GAL 4 expression. A. FoxP-GA L4 crossed with a membrane-bound green flourescent protein (UAS-CD8::GFP) revealed FoxP expressing cells in the third instar larval CNS, which was then stained with an antibody to GFP (green). B. FoxP antibody also labels FoxP expressing cells in the same brain (red). C. A pproximately identical overlap of the molecular tools used in $B$ and $C$ indicates that both are specific to FoxP cells. 


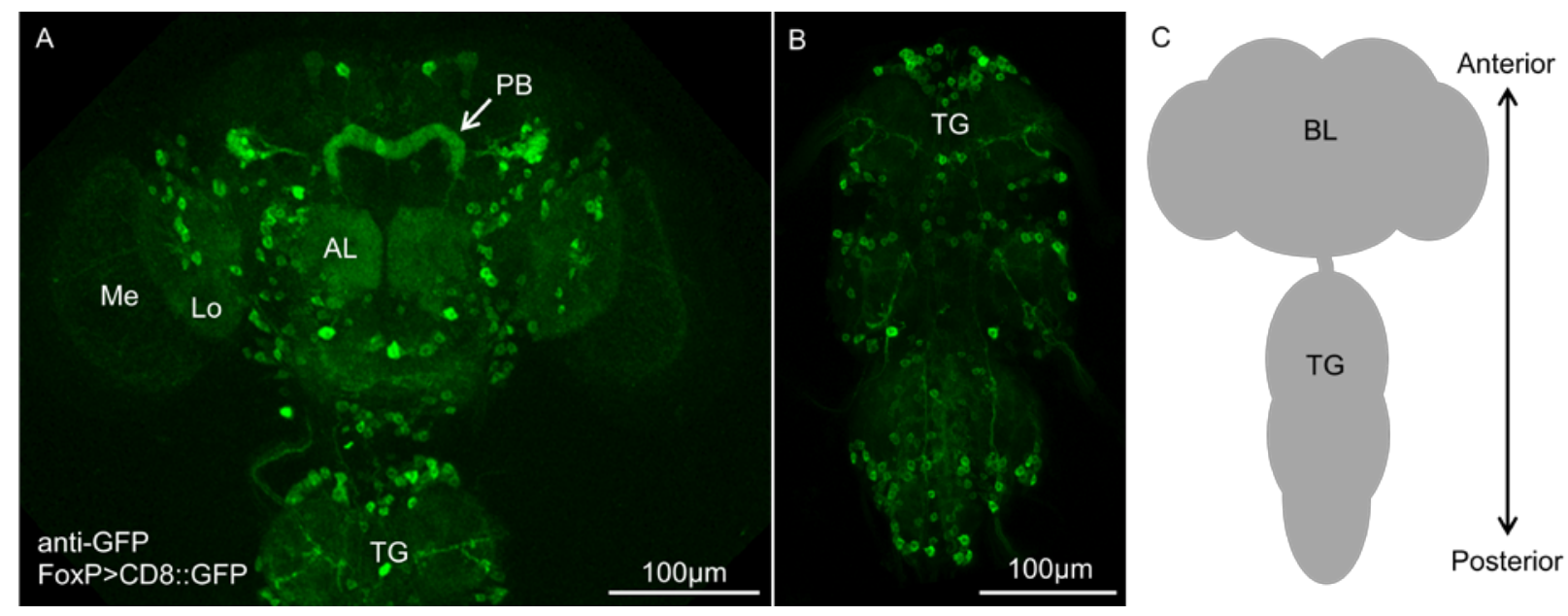

Figure 3. Expression pattern of FoxP in the adult brain. Expression of FoxP in the CNS is limited to a relatively small number of neurons in distinct clusters. A. A dult brain of a FoxP $>$ CD 8::GFP fly stained with anti-GFP shows strong labeling in the protocerebral bridge (white arrow) and other unidentified cell clusters. B. FoxP expressing cells are also widely distributed throughout the adult thoracic ganglion. C. Schematic of the adult CNS with symmetrical brain lobes and thoracic ganglion. $\mathrm{Me}=$ medulla, $\mathrm{LO}=$ lobula, $\mathrm{AL}=$ antennal lobe, $T G=$ thoracic ganglion . 
shaped protocerebral bridge (PB), which is a substructure in the central complex (CX). M any other small FoxP cell clusters are labeled throughout the adult CNS, but remain to be identified. Widely distributed expression is also seen throughout the thoracic ganglion in both larvae and adult. These expression patterns were consistently reliable across more than 30 adult and more than 20 larva brains, with approximately equal numbers of males and females from both FoxPnls::GFP and FoxP-CD8::GFP lines.

FoxP cells with nuclear localized GFP in adult flies were also stained with antibodies to the neuronal marker elav or the glial marker repo. FoxP expression appeared to be exclusively localized to neurons (Fig. 4), and not glia (Fig. 5).

\subsubsection{Q uantification of F oxP cells}

Estimated cell counts using FoxP-nls::GFP adult brains indicate a conservative approximation of $419 \pm 16$ neurons ( $n=6$ ), with no significant difference between the right and left lobes $(p=0.8499)$. We also observe no significant difference between males ( $413 \pm 17 ; n=3$ ) and females ( $424 \pm 17 ; n=3 ; p=0.2279$ )( Fig. 6). From qualitative visual inspection no obvious differences in expression pattern are noticeable between the sexes. M ean \pm SEM for each group.

\subsubsection{Eclosion timing}

When perturbing activity of FoxP expressing cells using a temperature sensitive shibire mutant, we see a dramatic affect on eclosion behavior. In earlier behavioral experiments we observed that Act5c $>$ RNAi males at $29^{\circ} \mathrm{C}$ all failed to eclose. FoxP $>$ UAS-Shits ${ }^{\text {kept }}$ at $30^{\circ} \mathrm{C}$ during pupation all failed to eclose $(0 \% ; n=30)$, whereas FoxP>UAS-Shits flies at room temperature nearly all successfully emerged as adults $(89 \% ; n=29)$. Importantly, FoxP $>$ UAS-

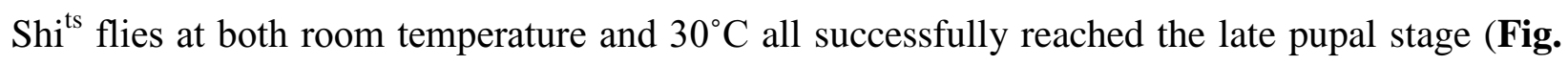
7). 


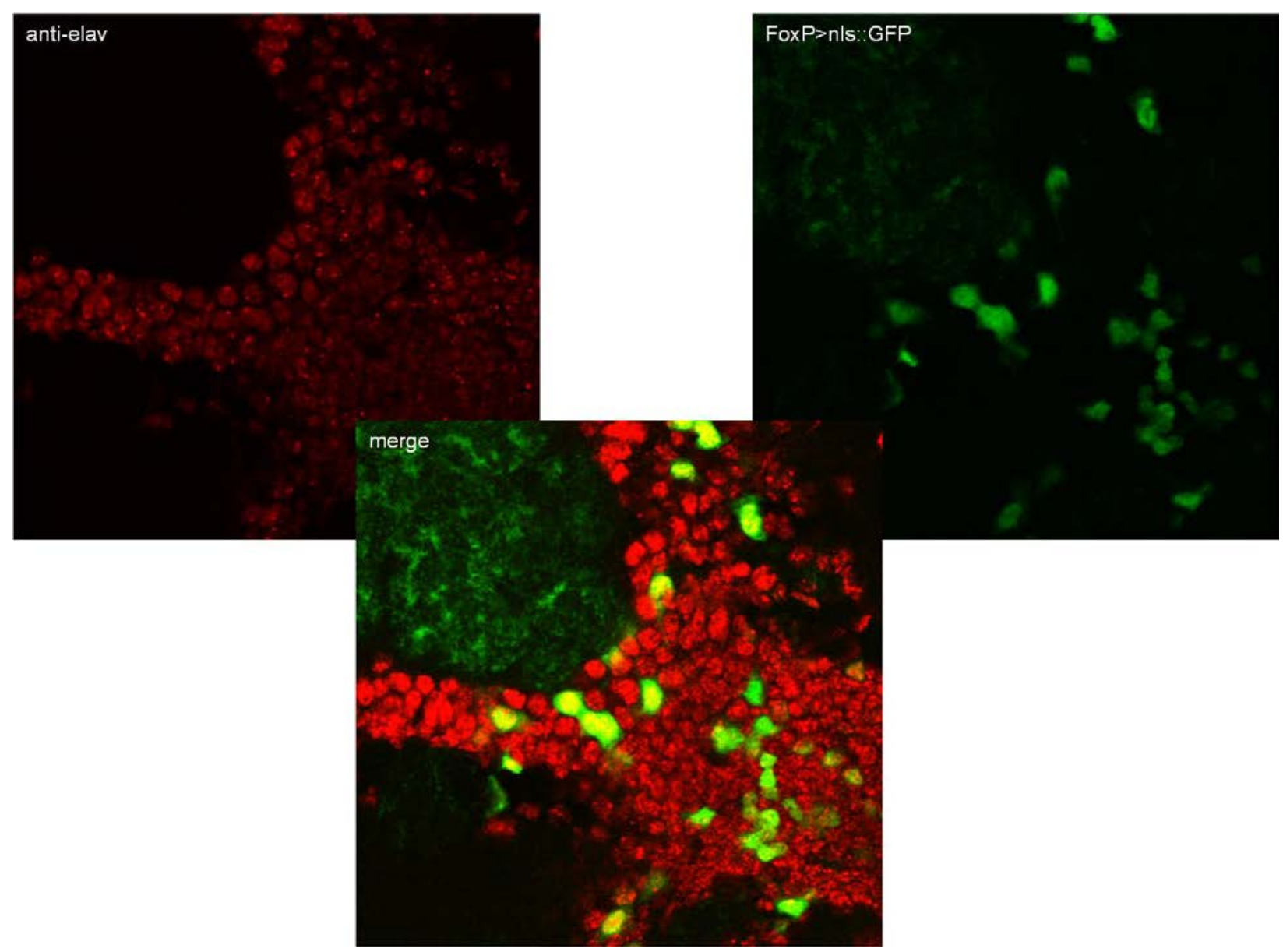

Figure 4. Confirming if FoxP is in neurons. Example of co-expression of FoxP $>n \mid s:$ GFP (green) and the neuronal marker elav (red) in a zoomed section of the adult brain. Overlap in the merged image indicates that these FoxP cells are neurons. 


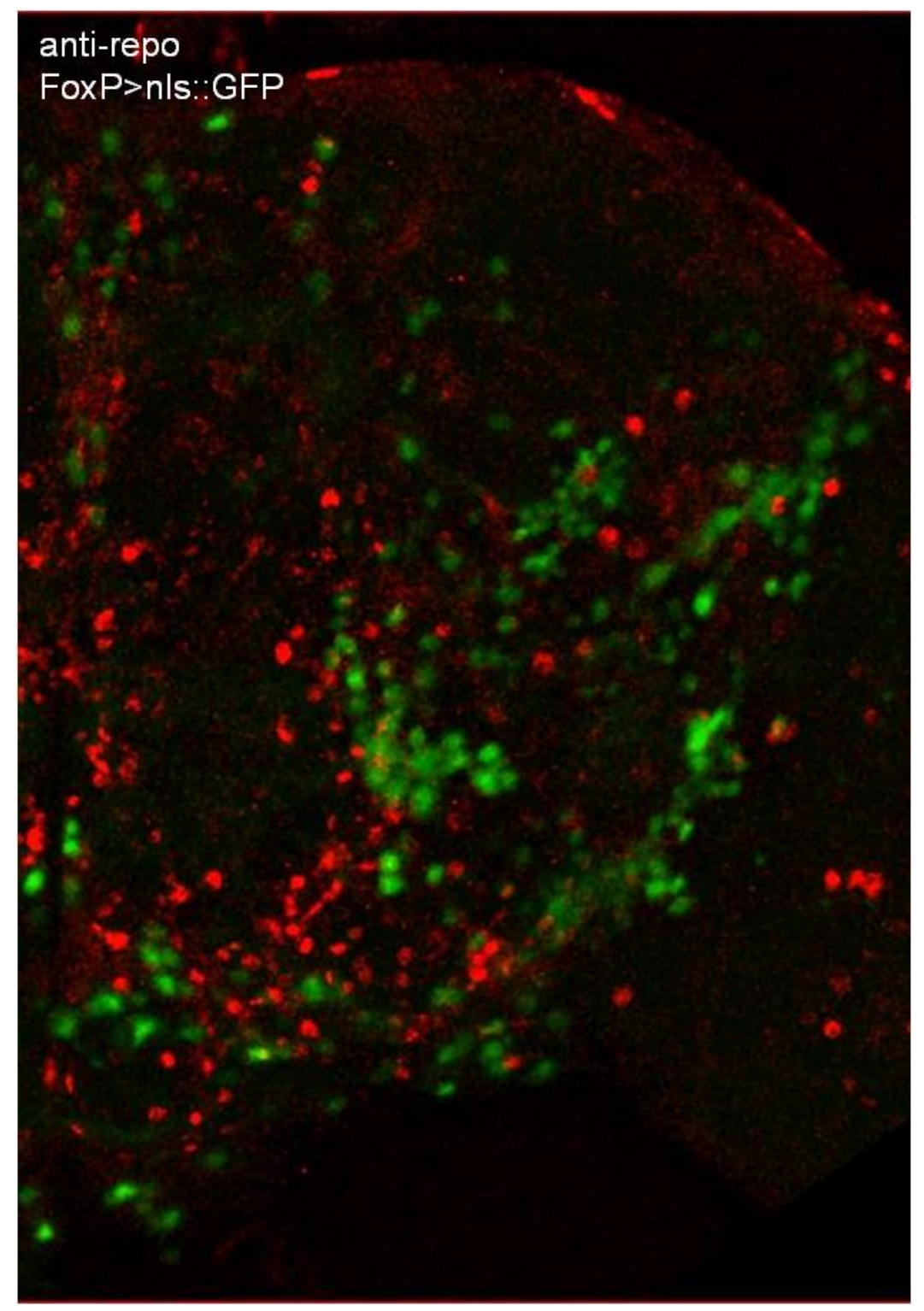

Figure 5. Confirming FoxP is not in glia. FoxP>nls::GFP (green) expression and antibody staining for the glial marker repo (red) in a single lobe of the adult brain. The absence of overlap indicates that FoxP is likely not expressed in glial cells. 

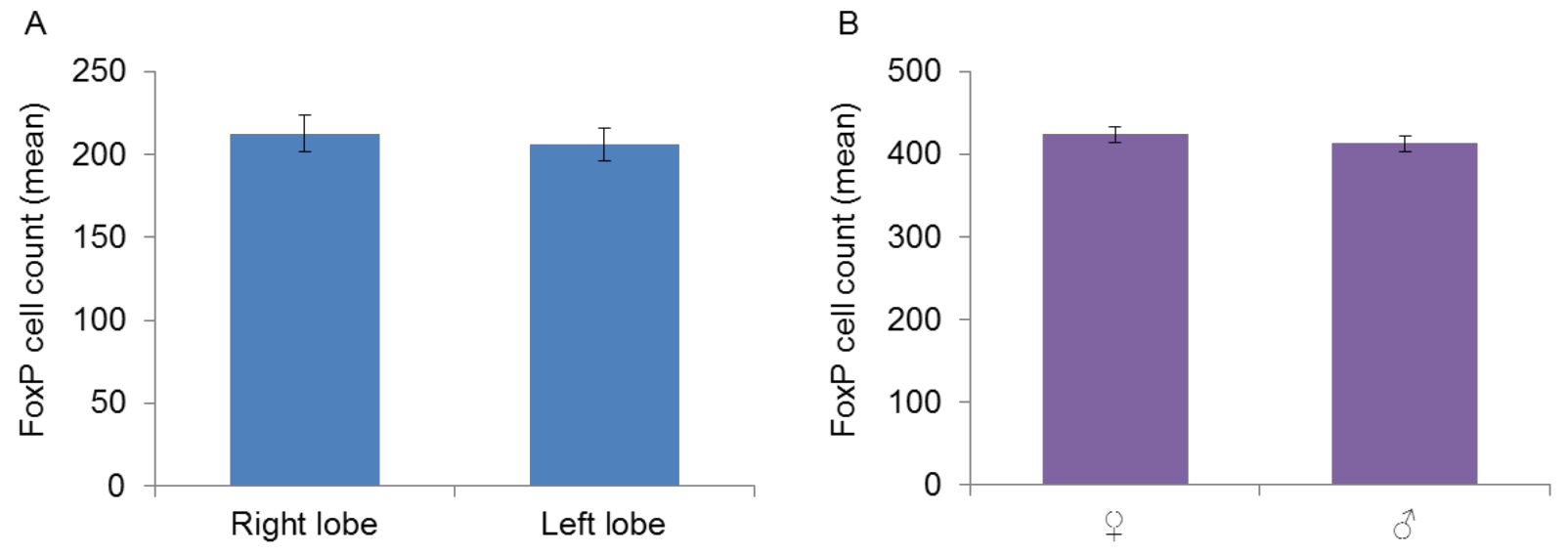

Figure 6. FoxP cell counts in FoxP>nls: :GFP adult brains. A. No significant difference in number of FoxP expressing cells is seen between the two brain lobes ( $n=6$ each; $p=0.8499$ ). B. No significant difference is seen in whole brain FoxP cell counts between males and females $(n=3$ each; $p=0.2279)$. M ean \pm SEM . 


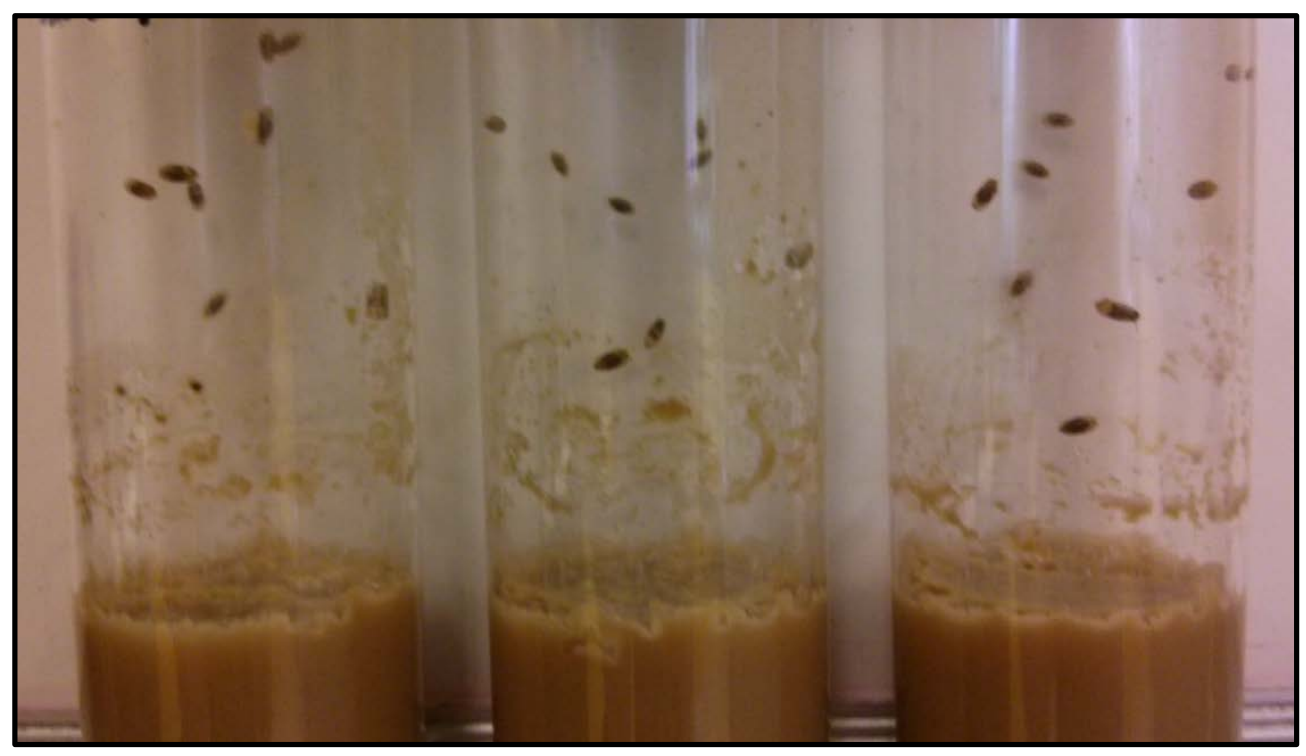

Figure 7. Disruption of FoxP neurons during pupation prevents eclosion. Examples of vials of FoxP $>\mathrm{Shi}^{\text {ts }}$ which when placed in a $30^{\circ} \mathrm{C}$ incubator during pupation all reach the late dark pupal stage but fail to eclose. Dark ovals are occupied pupal cases positioned on the walls of a vial with fly food media (light brown substance). 


\subsection{Discussion}

A s in vertebrates, FoxP is strongly expressed in a variety of regions in the brain. In larvae and adult we observed a relatively small number of neurons expressing FoxP, but distributed throughout a wide area of the CNS. This is similar to the expression pattern seen with in situ hybridization in the honeybee Apis mellifera (K iya et al. 2008). In adults some of these neurons are organized in distinct clusters, suggesting that FoxP may have direct behavioral effects though a specific identifiable motor-related network. Notably, FoxP+ neurons appear to send axonal projections within the distinctive protocerebral bridge (PB) structure, which is part of the central complex (CX). FoxP-CD 8::GFP flies show distinct external bilateral clusters that are potentially projecting into the PB. This is similar to recent findings of late-born external projecting neural lineages into the PB (Riebli et al. 2013). FoxP may be responsible for development of specific subsets of these neurons. A recently constructed wiring diagram of the PB (Lin et al. 2013) as well as establishment of the developmental origins of CX neurons ( $Y$ ang et al. 2013), may allow for future studies to determine the exact FoxP neurons which contribute to their PB expression. The function of the PB itself has not been extensively studied, although the CX as a whole is thought to be involved in higher locomotion control (Strauss 2002) and has been implicated in courtship song production in both Drosophila and grasshoppers (Popov et al. 2003; Heinrich et al. 2012). Some work has specifically examined the role of the PB using mutants with structural defects specific to the bridge - early studies with the no-bridge mutant indicate deficits in learning, walking speed, and courtship (B ouhouche et al. 1993; Strauss et al. 1992; Hall 1994). A more recent study implicated this structure in sensorimotor integration as demonstrated in a gap crossing assay. Two types of bridge mutants showed deficits in correct aiming when attempting gap crossing (Triphan et al. 2010). Although some of these deficits are consistent with our 
results, it is not possible to know at this point if the behavioral deficits we see in FoxP knockdown animals are due to disturbed activity in the PB or in one of the other unidentified FoxP expressing neuronal clusters, but it is an intriguing possibility that the role played by FoxP in motor coordination may be related to its expression in this part of the CX. Importantly, the CX has been compared to the vertebrate basal ganglia and the PB to the striatum (Strausfeld \& Hirth 2013), which in vertebrates is known to be important for motor coordination and shows strong FoxP2 expression (Lai et al. 2003; Ferland et al. 2003). Our work further contributes to the possibility of a deep homology in brain structure and function of the insect $C X$ and vertebrate basal ganglia.

Somew hat unexpectedly, considering the sex-specific effects on behavior, no obvious sex differences was noticeable at the morphological level, both in terms of expression pattern and cell counts in adult brains. The behavioral difference in flies could be due to undetected subtle variations in neuronal populations expressing FoxP between the sexes. Alternatively, the sex difference could be due to as yet unknown gender specific splice variants, as is the case with the well-studied Drosophila fruitless transcription factor (Dauwalder 2011). If the overall structure of the circuit is the same, it may be at the molecular level in that FoxP expression level or transcriptional targets vary between the sexes.

Suppression of activity in FoxP expressing neurons during pupation impaired eclosion behavior, where animals reached a late pupal stage but were unable to emerge as adults. The potentially less severe partial knockdown of FoxP expression in development with $\mathrm{Act} 5 \mathrm{c}>\mathrm{RNAi}$ at $29^{\circ} \mathrm{C}$ exhibits the sex-specific effect on eclosion as seen with other behaviors, but disruption of neural activity in FoxP>UAS-Shits flies affects both genders equally. The level of FoxP needed in development may be flexible or dose-dependent, but functional FoxP neurons are crucial for 
eclosion behavior. Given the motor impairments described in the previous chapter, a likely explanation is that these flies simply lack the motor ability to emerge from the pupal case. The fact that the larvae are able to successfully grow and pupate indicates that FoxP plays a less important role in earlier stages of development. From this, a tentative hypothesis may be that FoxP is performing a vital function in neural development during pupation - a time at which the nervous system has a major transition between larva and adult forms. Potentially supporting this is the known peak in FoxP expression at the mid-pupal stage (Graveley et al. 2011).

In conclusion, several intriguing parallels between FoxP and FoxP2 have emerged from this work, such as behavioral changes in the form of disrupted coordination of movement due to reducing FoxP expression in development or inhibiting activity in FoxP expressing cells. A lso correlated is the fact that FoxP expression is in select regions of the brain throughout development, including a subsection of an insect brain area known to be important for motor skills (the central complex) which has been proposed to be homologous to corresponding vertebrate motor control brain regions (the basal ganglia) (Strausfeld \& Hirth 2013). FoxP also appears to be playing a role in CNS development, with a particularly strong effect within the transition from larval to adult nervous systems during pupation. This work is far from complete but leads to a wide range of possibilities for further exploration of this gene in flies, possible identification of details pertaining to FoxP2, and insights into the evolutionary conservation of the developmental foundations of motor coordination. 


\section{REFERENCES}

Bouhouche, A., V aysse, G. \& Corbière, M., 1993. Immunocytochemical and learning studies of a Drosophila melanogaster neurological mutant, no-bridgeK S49 as an approach to the possible role of the central complex. J ournal of neurogenetics, 9(2), pp.105-21.

Carrillo, G.D. \& Doupe, A.J., 2004. Is the songbird A rea X striatal, pallidal, or both? An anatomical study. The J ournal of comparative neurology, 473(3), pp.415-37.

Dauwalder, B., 2011. The roles of fruitless and doublesex in the control of male courtship. International review of neurobiology, 99, pp.87-105.

Dietzl, G. et al., 2007. A genome-wide transgenic R NA i library for conditional gene inactivation in Drosophila. Nature, 448(7150), pp.151-6.

Enard, W. et al., 2009. A humanized version of Foxp2 affects cortico-basal ganglia circuits in mice. Cell, 137(5), pp.961-71.

Ferland, R.J. et al., 2003. Characterization of Foxp2 and Foxp1 mRNA and protein in the developing and mature brain. The J ournal of comparative neurology, 460(2), pp.266-79.

Fisher, S.E. \& Scharff, C., 2009. FOXP2 as a molecular window into speech and language. Trends in genetics/: TIG , 25(4), pp.166-77.

French, C.A. et al., 2012. An aetiological Foxp2 mutation causes aberrant striatal activity and alters plasticity during skill learning. M olecular psychiatry, 17(11), pp.1077-85.

Graham, S. a \& Fisher, S.E., 2012. Decoding the genetics of speech and language. Current opinion in neurobiology, pp.1-9.

Graveley, B.R. et al., 2011. The developmental transcriptome of Drosophila melanogaster. Nature, 471(7339), pp.473-9.

Haesler, S. et al., 2007. Incomplete and inaccurate vocal imitation after knockdown of FoxP2 in songbird basal ganglia nucleus A rea X. PL oS biology, 5(12), p.e321.

Hall, J., 1994. The mating of a fly. Science, 264(5166), pp.1702-1714.

Heinrich, R., Kunst, M. \& Wirmer, A., 2012. Reproduction-related sound production of grasshoppers regulated by internal state and actual sensory environment. Frontiers in neuroscience, 6(J une), p.89.

Kiya, T., Itoh, Y . \& Kubo, T., 2008. Expression analysis of the FoxP homologue in the brain of the honeybee, A pis mellifera. Insect molecular biology, 17(1), pp.53-60. 
Lai, C.S. et al., 2001. A forkhead-domain gene is mutated in a severe speech and language disorder. Nature, 413(6855), pp.519-23.

Lai, C.S.L. et al., 2003. FOXP2 expression during brain development coincides with adult sites of pathology in a severe speech and language disorder. Brain/: a journal of neurology, 126(Pt 11), pp.2455-62.

Lee, H. \& Frasch, M., 2004. Survey of forkhead domain encoding genes in the Drosophila genome: Classification and embryonic expression patterns. Developmental dynamics/: an official publication of the American Association of Anatomists, 229(2), pp.357-66.

Liégeois, F. et al., 2003. Language fM RI abnormalities associated with FOXP2 gene mutation. Nature neuroscience, 6(11), pp.1230-7.

Lin, C.-Y . et al., 2013. A comprehensive wiring diagram of the protocerebral bridge for visual information processing in the Drosophila brain. Cell reports, 3(5), pp.1739-53.

Loveall, B.J. \& Deitcher, D.L., 2010. The essential role of bursicon during Drosophila development. BMC developmental biology, 10, p.92.

Popov, A. V et al., 2003. The role of central parts of the brain in the control of sound production during courtship in Drosophila melanogaster. Neuroscience and behavioral physiology, 33(1), pp.53-65.

Reimers-Kipping, S. et al., 2010. Humanized Foxp2 specifically affects cortico-basal ganglia circuits. Neuroscience, pp.1-10.

Riebli, N., Viktorin, G. \& Reichert, H., 2013. Early-born neurons in type II neuroblast lineages establish a larval primordium and integrate into adult circuitry during central complex development in Drosophila. Neural development, 8(1), p.6.

Risinger, C. et al., 1997. Complex gene organization of synaptic protein SNA P-25 in Drosophila melanogaster. G ene, 194(2), pp.169-77.

Scharff, C. \& Petri, J., 2011. Evo-devo, deep homology and FoxP2: implications for the evolution of speech and language. Philosophical Transactions of the Royal Society B: Biological Sciences, 366(1574), pp.2124-2140.

Strausfeld, N.J . \& Hirth, F., 2013. Deep homology of arthropod central complex and vertebrate basal ganglia. Science (N ew York, N.Y.), 340(6129), pp.157-61.

Strauss, R. et al., 1992. No-bridge of Drosophila melanogaster: portrait of a structural brain mutant of the central complex. J ournal of neurogenetics, 8(3), pp.125-55.

Strauss, R., 2002. The central complex and the genetic dissection of locomotor behaviour. Current opinion in neurobiology, 12(6), pp.633-8. 
Tomblin, J.B. et al., 1997. Prevalence of specific language impairment in kindergarten children. J ournal of speech, language, and hearing research/: J SL HR, 40(6), pp.1245-60.

Triphan, T. et al., 2010. Visual targeting of motor actions in climbing Drosophila. Current biologyl: CB, 20(7), pp.663-8.

V argha-K hadem, F., W atkins, K. \& CJ, 1998. Neural basis of an inherited speech and language disorder. PNAS, 95(October), pp.12695-12700.

Watkins, K. \& V argha-K hadem, F., 2002. M RI analysis of an inherited speech and language disorder: structural brain abnormalities. Brain, 125, pp.465-478.

Yang, J.S. et al., 2013. Diverse neuronal lineages make stereotyped contributions to the Drosophila locomotor control center, the central complex. The Journal of comparative neurology, 521(12), pp.2645-62, Spc1. 


\section{CHAPTER 4}

\section{SUMMARY AND FUTURE DIRECTIONS}

\subsection{B rief overview}

In the previous chapters I used a reverse genetics approach to determine the function of the previously unexplored Drosophila melanogaster FoxP - with the goal of identifying possible similarities between it and the homologous human FOX P2 speech and language gene. A variety of genetic, molecular, and behavior techniques used to explore functional properties of FoxP revealed the behavioral importance, expression pattern, and critical timing of this gene in the nervous system.

\subsection{Characterizing the importance of Drosophila F oxP on fly behavior}

The questions addressed in Chapter 2 stem from the prediction that fly FoxP and human FOXP2 may both be involved in complex motor control. To test this we utilized techniques that reduced FoxP expression throughout development and disrupted FoxP expressing neurons in adults and tested effects on behavior.

\subsubsection{Knockdown of $\mathrm{F}$ oxP expression during development}

In order to determine the effect of FoxP on behavior, we used targeted RNA interference to partially reduce FoxP expression throughout development and tested effects on several behaviors, including courtship, song production, flight, and overall walking activity level. All of these behaviors were impaired by FoxP reduction, indicating a developmental role for this gene 
in neurons needed for coordinated motor control. Unexpectedly we also observed a sex-specific difference in the majority of these behaviors, with males more strongly impaired than females.

\subsubsection{Acute disruption of F oxP expressing neurons in adults}

Using a temperature sensitive shibire line to disrupt neurotransmission in adult FoxP expressing neurons allowed for more precise manipulation of the FoxP "network" on behavior. A ffected flies exhibited dramatic uncoordinated spastic movement and reduced walking activity, providing further support for a role of FoxP in motor coordination.

\subsection{Determining the location and timing of $F$ oxP action}

As a means of dissecting the underlying mechanisms of action of FoxP on behavior, it was necessary to localize FoxP in the nervous system as well as begin to define a critical period of FoxP neuronal activity in development. To accomplish this we created tools to visualize FoxP expression in the CNS and identified when in development this expression becomes essential for behavior.

\subsubsection{Expression pattern of $F$ oxP in the nervous system}

We created both a FoxP specific antibody and a FoxP-GA L4 transgenic line to visualize the location of FoxP expressing cells in the brain. The FoxP-GA L4 combined with a UAS-GFP line provided bright fluorescent labeling of FoxP cells. This identified a relatively small number of neurons symmetrically expressed in the brain lobes with no obvious difference between males

and females. Strong expression was seen in an area of the insect central complex known as the 
protocerebral bridge. This structure may be a link between FoxP expression and behavior, as it is a region previously implicated in motor control.

\subsubsection{Timing}

As evident from Chapter 2, FoxP neurons are essential for motor coordination in adults, but when in development is FoxP expression acting on these neurons? Initial observation that strong FoxP RNAi knockdown prevented eclosion, combined with data from other studies showing FoxP peak expression during the mid-pupal stage, prompted us to examined the importance of this time point more closely. We used the FoxP>UAS-Shits line to disrupt neurotransmission in FoxP neurons specifically during pupation and observed effects on eclosion behavior. This perturbation prevented eclosion in $100 \%$ of animals but allowed them all to reach late pupal stage normally. This suggests that FoxP expression may be playing a key role in the redevelopment of the nervous system from larvae to adult.

\subsection{F uture directions}

As this work is the first functional study of FoxP in flies, much remains to be explored. An obvious question to address next would be identifying cell types which make up the FoxP network and how these are connected to behavior. This could be done by looking for colocalization of FoxP with a variety of neurotransmitter cell types. If FoxP neurons are comprised of particular neuron classes, then knockdown with RNAi or conditional manipulations in subsets of these could potentially provide a method of determining which FoxP neurons are most important for behavior. It would be of great interest to identify properties of the neurons projecting through the PB and manipulate these specifically. 
Generation of FoxP mutants would provide highly desirable tools to complement the RNA i knockdown studies, especially if located in the conserved portion of the forkhead domain, or with a mutation in the same residue as the KE family, as was done in mice (Fujita et al. 2008). It would also be helpful to generate mutations or RNAi specific to each FoxP isoform to elucidate their specific functions. A mostly specific PB mutant such as nob (Strauss et al. 1992) could help determine how this area of FoxP expression is tied to behavior, although it may not be the only structure involved in the FoxP phenotype. It would also be difficult to interpret similarities in phenotype given that the PB is al ready known to affect locomotion.

Although this work established the pupal stage as a necessary time for normal FoxP cell function, a closer look at all developmental time points is essential. FoxP may play multiple roles at different stages. An intriguing result is the observation of abnormal larva crawling in knockdown animals. M easurement of speed and turning rate would perhaps be more informative than overall distance travelled. A dapting the larva locomotion assay to an incubator setting for the FoxP $>S_{\text {hits }}{ }^{\text {ls }}$ line would answer an important question about the effects of FoxP neurons in larvae and at finer time scales in pupae. Timing gradation of FoxP knockdown is also possible with tools which allow for drug or temperature induced knockdown at specified time points. This would be useful to determine if FoxP expression still plays an active role in adulthood.

This work explored deficits in a broad range of behaviors, but further work should explore the details at a finer level. A closer examination of some aspects of these behaviors may be able to determine the degree and specificity of coordination issues, especially with the FoxP $>$ Shits line if specific behavioral assays are adapted to an incubator. With information from this it may be possible to determine a more precise function of FoxP neurons, such as whether they are modulating a specific CPG network. 
Sex-specific behavioral effects in flies should provide incentive to look more closely at potential sex differences in vertebrates as well. There are many possible ways from which the difference can originate. It may be variations in when, where, or how much FoxP is expressed between genders. Although no obvious difference was visually noticeable, our method of cell counting would likely miss small changes in cell number or position. It should be possible to compare expression level variation between the sexes at different life stages, although these differences may only become relevant after differentiation of sex specific behaviors. FoxP knockdown larvae did show a difference in crawling behavior, it was not significantly different between the males and females.

Since FoxP is acting as a transcription factor, it is likely influencing development through regulating other genes. There are a few different ways to investigate this. One approach is to determine which genes are regulated in the fly by FoxP by chromatin immunoprecipitation or by RNA -seq in order to identify candidate genes to explore. But another approach is to leverage the work done in mammals and examine the highly conserved fly homologs of mammalian genes already known to be regulated by FoxP2 (V ernes et al. 2007; Spiteri et al. 2007; R oll et al. 2010). Perturbation and testing of these target genes may provide valuable clues to the development of FoxP expressing cells and potential insight into FoxP2 function.

The insect CX and vertebrate BG are related in genetic, developmental, and functional ways (Strausfeld \& Hirth 2013). A recent paper even compared the complexity of courtship to human grammar (Stoop et al. 2013). A lthough Drosophila melanogaster is the most established insect genetic model, conservation of FoxP properties should also be explored between other insects. Targeted sequencing and annotation of this gene in a variety of invertebrate species may contribute to understanding the function and evolution of FoxPs in vertebrates as well. 
The work presented in this dissertation has described functional properties of a previously unexplored fly gene, provided possible insights into a critical motor control structure in insects, and implied a deep homology for a role in motor coordination between a fly and human speech gene. Is there any real meaning to similarity between FoxP and FoxP2, especially given the many differences between insect and human brains? We won't know until a discovery in one sheds light on the story in the other, but it has certainly happened before that something we've learned in flies has taught us something about ourselves (Lehmann et al. 2003). 


\section{REFERENCES}

Fujita, E. et al., 2008. Ultrasonic vocalization impairment of Foxp2 (R552H) knockin mice related to speech-language disorder and abnormality of Purkinje cells. Proceedings of the National Academy of Sciences of the U nited States of America, 105(8), pp.3117-22.

Lehmann, 0.J. et al., 2003. Fox's in development and disease. Trends in genetics/: TIG, 19(6), pp.339-44.

Roll, P. et al., 2010. Molecular networks implicated in speech-related disorders: FOXP2 regulates the SR PX 2/uPA R complex. Human molecular genetics, 19(24), pp.4848-60.

Spiteri, E. et al., 2007. Identification of the transcriptional targets of FOXP2, a gene linked to speech and language, in developing human brain. American journal of human genetics, 81(6), pp.1144-57.

Stoop, Ruedi et al., 2013. A t Grammatical Faculty of Language, Flies Outsmart M en. PloS one, $8(8)$, pp.1-5.

Strausfeld, N.J . \& Hirth, F., 2013. Deep homology of arthropod central complex and vertebrate basal ganglia. Science (N ew York, N.Y.), 340(6129), pp.157-61.

Strauss, R. et al., 1992. No-bridge of Drosophila melanogaster: portrait of a structural brain mutant of the central complex. J ournal of neurogenetics, 8(3), pp.125-55.

Vernes, S.C. et al., 2007. High-throughput analysis of promoter occupancy reveals direct neural targets of FOX P2, a gene mutated in speech and language disorders. American journal of human genetics, 81(6), pp.1232-50. 